\title{
Inflammation in Ischemic Stroke Subtypes
}

\author{
Antonino Tuttolomondo ${ }^{1 *}$, Domenico Di Raimondo ${ }^{1}$, Rosaria Pecoraro ${ }^{1}$, Valentina $\mathrm{Arnao}^{2}$, Antonio Pinto ${ }^{1}$ \\ and Giuseppe Licata ${ }^{1}$
}

${ }^{1}$ Dipartimento Biomedico di Medicina Interna e Specialistica, Università degli Studi di Palermo (Italy); ${ }^{2}$ Dipartimento di Biopatologia e Neuroscienze Cliniche, Università degli Studi di Palermo, Italy

\begin{abstract}
Determining the cause of stroke does influence choices for management. categorization of subtypes of ischemic stroke has had considerable study, but definitions are hard to formulate and their application for diagnosis in an individual patient is often problematic. Cerebral ischemia initiates a complex cascade of events at genomic, molecular, and cellular levels, and inflammation is important in this cascade. In 1993 for For the Trial of Org 10172 in Acute Stroke Treatment (TOAST), Adams et al] conducted a placebo-controlled, randomized, blinded study of the low-molecular-weight heparinoid given to patients within 24 hours after stroke and developed a system for diagnosis of subtype of ischemic stroke that uses components of existing diagnostic schemes. The type of acute ischemic stroke was classified according to the TOAST classification: 1) Large Artery AtheroSclerosis (LAAS); 2) CardioEmbolic Infarct (CEI); 3) LACunar infarct (LAC); 4) stroke of Other Determined Etiology (ODE); 5) stroke of UnDetermined Etiology (UDE) (see Fig. (1)). On the basis of pathophysiologic differences of each stroke subtype it's possible to hypothesize a different pattern of immuno-inflammatory activation in relation of ischemic stroke subtype. A nonspecific systemic inflammatory response occurs after both ischemic and hemorrhagic stroke, either as part of the process of brain damage or in response to complications such as deep venous thrombosis. Several studies have reported that higher levels of inflammatory markers such as C-reactive protein (CRP) and interleukin-6 (IL-6) are associated with worse outcome after ischemic stroke.

Our group reported that patients with cardioembolic subtype showed significantly higher median plasma levels of TNF- $\alpha$, IL-6, IL-1 $\beta$ whereas the lacunar subtype showed significantly lower median plasma levels of TNF- $\alpha$, IL- 6 and IL-1 $\beta$. Our findings underlined the significant association was noted between the severity of neurological deficit at admission, the diagnostic subtype and some inflammatory variables.
\end{abstract}

Keywords: Stroke, inflammation, cytokines.

\section{INTRODUCTION}

The two broad categories of stroke, hemorrhage and ischemia, are diametrically opposite conditions: hemorrhage is characterized by too much blood within the closed cranial cavity, while ischemia is characterized by too little blood to supply an adequate amount of oxygen and nutrients to a part of the brain [1]. Each of these categories can be divided into subtypes that have somewhat different causes, clinical pictures, clinical courses, outcomes, and treatment strategies. As an example, intracranial hemorrhage can be caused by intracerebral hemorrhage (ICH), also called parenchymal hemorrhage, which involves bleeding directly into brain tissue, and subarachnoid hemorrhage (SAH), which involves bleeding into the cerebrospinal fluid that surrounds the brain and spinal cord.

Stroke subtyping can have different purposes, e.g. describing patients' characteristics in a clinical trial, grouping patients in an epidemiological study, careful phenotyping of patients in a genetic study, and classifying patients for therapeutic decision-making in daily practice. The classification should distinguish between ischemic and hemorrhagic stroke, subarachnoid hemorrhage, cerebral venous thrombosis, and spinal cord stroke. Regarding the 4 main categories of etiologies of ischemic stroke (i. e. atherothrombotic, small vessel disease, cardioembolic, and other causes), the classification should reflect the most likely etiology without neglecting the vascular conditions that are also found (e. g. evidence of small vessel disease in the presence of severe large vessel obstructions). Phenotypes of large cohorts can also be characterized by surrogate markers or intermediate phenotypes (e.g. presence of internal carotid artery plaque, intima-media thickness of the common carotid artery, leukoaraiosis, microbleeds, or multiple lacunae). Parallel classifications (i. e. surrogate markers) may serve

*Address correspondence to this author at the Dipartimento Biomedico di Medicina Interna e Specialistica, Università degli Studi di Palermo, Italy;

E-mail: bruno.tuttolomondo@unipa.it as within-study abnormalities to support research findings. Our group reported

Until recently, observational epidemiological studies of stroke were based mainly on pathological types of stroke. Many of these studies, which adopted near "ideal" methods, 5-17 had, in general, limited access to both the clinical expertise and the technologies (eg, carotid ultrasound, CT or MR imaging, and echocardiography) to perform a valid etiologic subtype classification. Increasingly, epidemiology is following clinical practice and not only considers all strokes together as the necessary first step but divides them into the 3 pathological types and then into various subtypes as well.

Determining the cause of stroke does influence choices for management. ategorization of subtypes of ischemic stroke has had considerable study, but definitions are hard to formulate and their application for diagnosis in an individual patient is often problematic. In the past, classifications have been based primarily on risk factor profiles, clinical features of the stroke, and the findings on brain imaging studies Yet, clinical and brain imaging features overlap and are not specific for any particular subtype of ischemic stroke.

Cerebral ischemia initiates a complex cascade of events at genomic, molecular, and cellular levels, and inflammation is important in this cascade, both in the Central Nervous System (CNS) and in the periphery. The entire spectrum of inflammatory processes is likely to act in concert in stroke but cytokines are important mediators of stroke-induced immunological/inflammatory reaction which contributes to brain infarct progression as well as to the disease severity and outcome.

On the basis of pathophysiologic differences of each stroke subtype it's possible to hypothesize a different pattern of immunoinflammatory activation in relation of ischemic stroke subtype. 


\section{ISCHEMIC STROKE SUBTYPES}

Until recently, observational epidemiological studies of stroke were based mainly on pathological types of stroke. Many of these studies, which adopted near "ideal" methods, $[2,8]$ had, in general, limited access to both the clinical expertise and the technologies (eg, carotid ultrasound, CT or MR imaging, and echocardiography) to perform a valid etiologic subtype classification. Increasingly, epidemiology is following clinical practice and not only considers all strokes together as the necessary first step but divides them into the 3 pathological types and then into various subtypes as well.

Categorization of subtypes of ischemic stroke has had considerable study, but definitions are hard to formulate and their application for diagnosis in an individual patient is often problematic. In the past, classifications have been based primarily on risk factor profiles, clinical features of the stroke, and the findings on brain imaging studies (computed tomography [CT] or magnetic resonance imaging $[\mathrm{MRI}])$. Yet, clinical and brain imaging features overlap and are not specific for any particular subtype of ischemic stroke.

Bamford et a1. [9] recently reported that outcomes and likelihood of recurrent stroke differed markedly by stroke subtype; large hemispheric infarcts, usually resulting from occlusion of the internal carotid artery or proximal middle cerebral artery, had the worst prognosis.

These investigators classified the strokes based on clinical features that forecasted the size and site of the ischemic lesion, but they did not consider the potential etiology of the stroke. Other investigators have noted that the etiology of stroke does influence prognosis. Sacco et a1. [10] noted that mortality was higher among patients with large-artery atherosclerotic lesions than among patients with lacunes. Recurrent strokes are more likely among patients with cardioembolic stroke than among patients with stroke of other causes $[11,12]$. The 1-month mortality after cardioembolic stroke is also higher than that with strokes of other etiologies [13, 14].

In 1993 for For the Trial of Org 10172 in Acute Stroke Treatment (TOAST), Adams et al [15] conducted a placebo-controlled, randomized, blinded study of the low-molecular-weight heparinoid given to patients within 24 hours after stroke and developed a system for diagnosis of subtype of ischemic stroke that uses components of existing diagnostic schemes. The type of acute ischemic stroke was classified according to the TOAST classification: 1) Large Artery AtheroSclerosis (LAAS); 2) CardioEmbolic Infarct (CEI); 3) LACunar infarct (LAC); 4) stroke of Other Determined Etiology (ODE); 5) stroke of UnDetermined Etiology (UDE) [see Fig. (1)]

Large Artery AtheroSclerosis (LAAS): These patients will have clinical and brain imaging findings of either significant $(>50 \%)$ stenosis or occlusion of a major brain artery or branch cortical artery, presumably due to atherosclerosis. Clinical findings include those of cerebral cortical impairment (aphasia, neglect, restricted motor involvement, etc. ) or brain stem or cerebellar dysfunction. Cortical or cerebellar lesions and brain stem or subcortical hemispheric infarcts greater than $1.5 \mathrm{~cm}$ in diameter on CT or MRI are considered to be of potential large-artery atherosclerotic origin. Supportive evidence by duplex imaging or arteriography of a stenosis of greater than $50 \%$ of an appropriate intracranial or extracranial artery is needed. Diagnostic studies should exclude potential sources of cardiogenic embolism.

\section{CardioEmbolic Infarcts (CEI)}

This category includes patients with arterial occlusions presumably due to an embolus arising in the heart. Cardiac sources are divided into high-risk and medium-risk groups based on the evidence of their relative propensities for embolism. At least one cardiac source for an embolus must be identified for a possible or probable diagnosis of cardioembolic stroke. Clinical and brain imaging findings are similar to those described for large-artery atherosclerosis. Evidence of a previous TIA or stroke in more than one vascular territory or systemic embolism supports a clinical diagnosis of cardiogenic stroke. Potential large-artery atherosclerotic sources of thrombosis or embolism should be eliminated. A stroke in a patient with a medium-risk cardiac source of embolism and no other cause of stroke is classified as a possible cardioembolic stroke.

\section{LACunar Infarct (LAC)}

The patient should have one of the traditional clinical lacunar syndromes and should not have evidence of cerebral cortical dysfunction. A history of diabetes mellitus or hypertension supports the clinical diagnosis. The patient should also have a normal CT/MRI examination or a relevant brain stem or subcortical hemispheric lesion with a diameter of less than $1.5 \mathrm{~cm}$ demonstrated.

Stroke of Other Determined Etiology (ODE): This category includes patients with rare causes of stroke, such as non-atherosclerotic vasculopathies, hypercoagulable states, or hematologic disorders. Patients in this group should have clinical and CT or MRI findings of an acute ischemic stroke, regardless of the size or location. Diagnostic studies such as blood tests or arteriography should reveal one of these unusual causes of stroke. Cardiac sources of embolism and large-artery atherosclerosis should be excluded by other studies.

\section{Stroke of UnDetermined Etiology (UDE)}

In several instances, the cause of a stroke cannot be determined with any degree of confidence. Some patients will have no likely etiology determined despite an extensive evaluation. In others, no cause is found but the evaluation was cursory.

Because most etiologic diagnoses in stroke are not based on pathological confirmation and are thus presumptive, investigators are allowed to express their certainty by classifying the likelihood of diagnosis as probable or possible. This is an important attribute of the TOAST classification system because it permits more careful scrutiny of those cases in whomthe diagnosis is uncertain and may help any adjudication process. Any categorization of subtypes of ischemic stroke contains some mixture of patients with similar but not identical brain injuries or vascular pathologies. It also includes some degree of inflexibility, a feature that is in common with any system of grouping patients; for example, a physician cannot diagnose cardioembolism in a patient who has a suggestive history when the diagnostic studies do not substantiate the presence of heart disease.

Based on the experiences of several large singlecenter or multicenter demographic studies, the most common causes of stroke are large-artery atherosclerosis, cardioembolism, and small-artery occlusion $[16,17]$. Because these are the three largest groupings of ischemic stroke and because the prognoses of patients in these divisions differ, examining responses to acute treatment in these groups is clinically important.

On the basis of TOAST classification Kolominsky-Rabas [18] conducted a study to determine the incidence, recurrence, and longterm survival rates of ischemic stroke subtypes by a mechanismbased classification scheme (Trial of ORG 10172 in Acute Stroke Treatment, or TOAST). Authors identified all 583 residents of the city of Erlangen, Bavaria, Germany, with a first ischemic stroke between 1994 and 1998. Multiple overlapping sources of information were used to ensure completeness of case ascertainment. The cause of ischemic stroke was classified according to the TOAST criteria. Patients were followed up at 3 months and 1 and 2 years after stroke onset. In this study The age-standardized incidence rates for the European population (per 100 000) regarding ischemic stroke subtypes were as follows: cardioembolism, 30. $2(95 \% \mathrm{CI}$ 25. 6 to 35.7 ); small-artery occlusion, 25.8 (95\% CI 21.5 to 30 . 


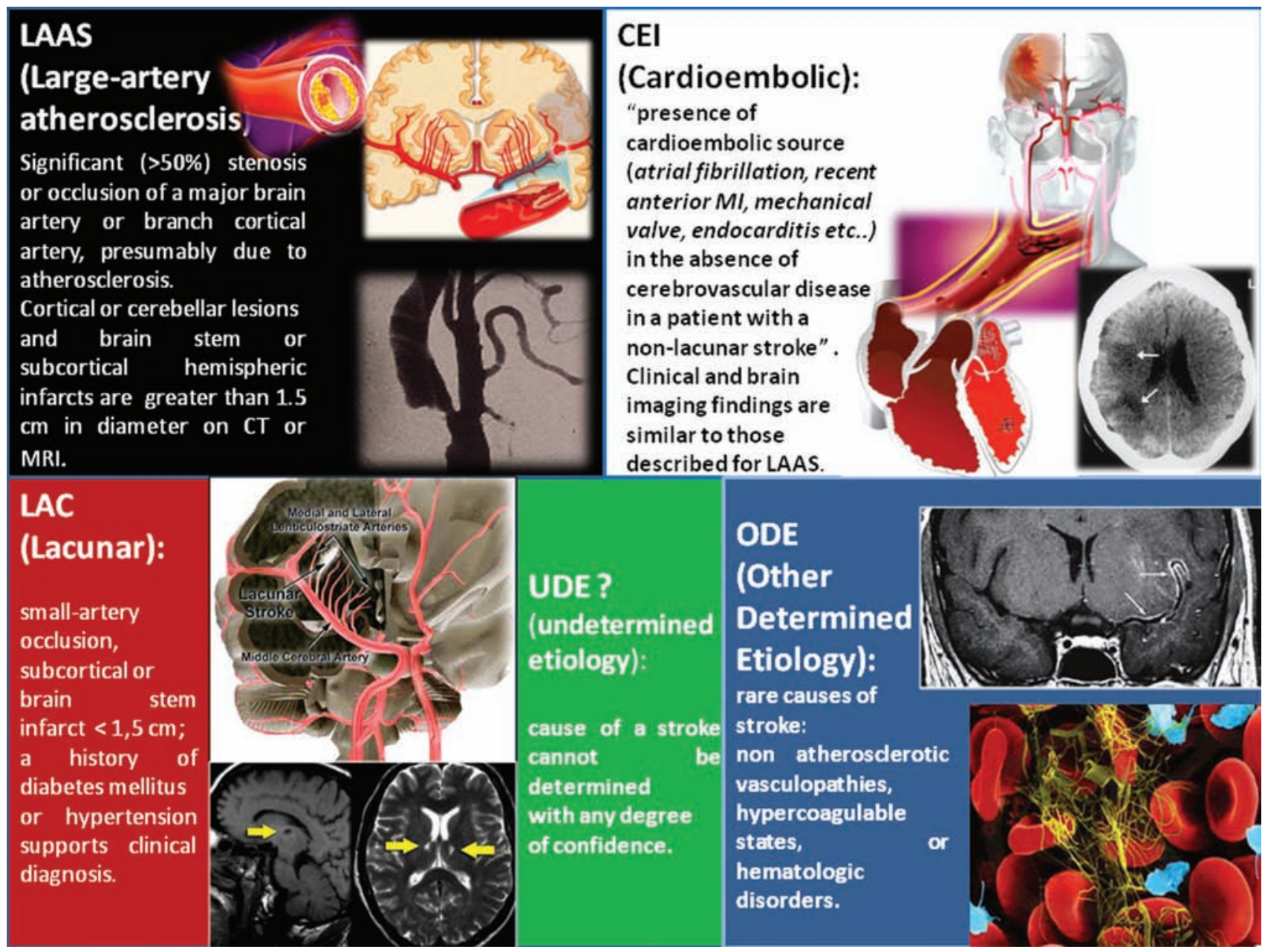

Fig. (1).

9); and large-artery atherosclerosis, 15. 3 (95\% CI 12 to 19. 3). When age-adjusted to the European population, the incidence rate for large-artery atherosclerosis was more than twice as high for men than for women (23. 6/100 000 versus 9. 2/100 000). Two years after onset, patients in the small-artery occlusion subgroup were 3 times more likely to be alive than those with cardioembolism. Ischemic stroke subtype according to the TOAST criteria was a significant predictor for long-term survival, whereas subtype was not a significant predictor of long-term recurrence up to 2 years, both before and after adjustment for age and sex.

This prospective study was the first population-based study on incidence, recurrence, and long-term survival of ischemic stroke subtypes with the TOAST criteria used as a mechanism-based subtype classification scheme. The present population-based study used widely accepted standardized methods of case ascertainment and provides for the first time basic epidemiological data on causes of ischemic stroke in a European population.

Petty et al. [19] conducted another population-based study on incidence and risk factors for ischemic stroke subtypes. Authors identified all 454 residents of Rochester, Minn, with a first ischemic stroke between 1985 and 1989 from the Rochester Epidemiology Project medical records linkage system. We used Stroke Data Bank criteria to assign infarct subtypes after reviewing medical records and brain imaging. We adjusted average annual incidence rates by age and sex to the US 1990 population and compared the ageadjusted frequency of stroke risk factors across ischemic stroke subtypes. This study reported age- and sex-adjusted incidence rates (per 100000 population) were as follows: large-vessel cervical or intracranial atherosclerosis with $>50 \%$ stenosis, 27 ; cardioembolic, 40; lacuna, 25; uncertain cause, 52; other or uncommon cause, 4. Sex differences in incidence rates were detected only for atherosclerosis with stenosis (47 [95\% CI, 34 to 61] for men; 12 [95\% CI, 7 to 17] for women). There was no difference in prior transient ischemic attack and hypertension among subtypes, and diabetes was not more common among patients with lacunar infarction than other common subtypes. The age-adjusted incidence rate of stroke due to stenosis of the large cervicocephalic vessels is nearly 4 times higher for men than for women. There is no association between preceding transient ischemic attack and stroke mechanism. Diabetes and hypertension are not more common among patients with lacunae. Age- and sex-adjusted incidence rates for ischemic stroke subtypes in this population can be compared with similarly determined rates from other populations.

Subtype-specific ischemic stroke incidence rates permit identification of racial and sex differences in stroke etiology. For example, our study documents that men have a 4 times greater ageadjusted incidence rate of ischemic stroke due to large-vessel atherosclerosis than women. This biological difference could more than adequately explain why carotid endarterectomy rates in the United States are $30 \%$ to $60 \%$ higher for men than for women $[20,21]$. Similarly, a comparison of our study and the study of the black population of metropolitan Cincinnati, [22] demonstrates that al- 
though black Americans have higher overall age- and sex-adjusted ischemic stroke incidence (246 per 100 000) compared with whites (147 per 100000 ), the incidence of stroke due to large-vessel atherosclerosis with stenosis is significantly greater among whites (27 per 100000$)$ than blacks (17 per 100000$)$. This difference cannot be attributed to a disparity in procedure rates because the same proportion (54\%) of patients in our study and in the study by Woo et al. received diagnostic tests to detect carotid stenosis. Clearly, biological differences in stroke mechanism as well as biological differences in the intracranial and extracranial distribution of atherosclerosis between blacks and whites [23, 24] could account for a significant portion of the 2- to 3-fold higher carotid endarterectomy rates reported for whites compared with blacks in Massachusetts, California, and the Veterans Affairs Medical Centers. 8, 13-15 Subtype-specific stroke incidence rates thus permit an informed and objective assessment of various hypotheses that have been proposed to explain race and sex differences in cerebral angiography and carotid endarterectomy rates in the United States [25-28].

Much of the heterogeneity in stroke can be related to the prevalence of risk factors, but some populations have a higher stroke incidence than would be predicted from risk factor levels. Hypertension, including borderline hypertension, is probably the most important stroke risk factor based on degree of risk and prevalence. However, cardiac morbidity, cigarette smoking, diabetes, physical inactivity, and high levels of alcohol consumption are also strongly related to stroke risk. High levels of blood cholesterol and homocysteine may also increase stroke risk. Mortality after stroke is highest within the first 30 days but remains elevated to a degree that depends on the presenting stroke syndrome, stroke subtype, and other co-morbidities. Lacunar strokes have the best short- and longterm prognoses. Strokes due to large-vessel atherosclerosis frequently worsen; these and cardioembolic strokes have the poorest long-term prognosis. The risk for recurrence is also highest within 30 days after a first stroke, depending on the type of infarct, history of hypertension, and blood glucose levels on admission. Predictors of late recurrence include cardiac disease, hypertension, and heavy alcohol use. Only about half of stroke survivors are independent 6 months after a stroke, and quality of life is decreased. Understanding factors that predispose to stroke and determine its outcome will help in the design of acute stroke trials and in prevention programs.

Incidence of ischaemic stroke subtypes, classified by cause, seems to vary between communities. Recently Lavados et al. [29] aimed to prospectively ascertain the incidence of first-ever ischaemic stroke in a predominantly Hispanic-Mestizo population in the northern desertic region of Chile. Between July, 2000, and June, 2002 , all patients with possible stroke or transient ischaemic attacks were identified from multiple overlapping sources and were rapidly assessed by two field neurologists. All identified patients were diagnosed by at least two stroke neurologists according to Trial of Org 10172 in Acute Stroke Treatment (TOAST) definitions and were followed up at 6 months. Annual incidence rates were age adjusted to WHO, European, and US populations by the direct method to allow comparisons. Authors identified a total of 239 ischaemic strokes, of which $185(77 \%)$ were first-ever cases. 151 $(82 \%)$ patients were hospitalised, of whom only $70(38 \%)$ were assessed within $6 \mathrm{~h}$ of symptom onset. The mean age of patients was 66. 4 years (SD 14.9) and 56\% were men. The crude annual incidence rates (per 100 000) according to stroke subtype were: cardioembolic, 9. 3; large-artery disease, 2. 0; small-vessel disease, 15. 8; other determined cause, 0. 2; and undetermined cause, 17. 4. Hypertension was the most common cardiovascular risk factor in all subtypes and atrial fibrillation was the most common cause of cardioembolic stroke. Case fatality at 30 days was highest in cardioembolic strokes $(28 \%)$ and lowest in small-vessel disease $(0 \%)$. Dependency or death at 6 months was also highest in cardioembolic strokes $(62 \%)$ and lowest in small-vessel disease $(21 \%)$. Incidence and prognosis of small vessel and cardioembolic infarction was similar to that in other populations and incidence of large-artery atherothrombotic infarction was lower than in most previous reports. Hypertension and atrial fibrillation were the most common risk factor and cause, respectively, of ischemic stroke in this population. These findings should help the national stroke programme in the prevention of cardioembolic stroke, increase access to specialists and acute brain imaging and vascular studies, and improve stroke care.

\section{INFLAMMATORY MARKERS IN ACUTE ISCHEMIC STROKE}

A nonspecific systemic inflammatory response occurs after both ischemic and hemorrhagic stroke, either as part of the process of brain damage or in response to complications such as deep venous thrombosis. Several studies have reported that higher levels of inflammatory markers such as C-reactive protein (CRP) and interleukin-6 (IL-6) are associated with worse outcome after both ischemic [30] and hemorrhagic [31, 32] strokes. However, these studies often had methodological weaknesses, chiefly that they were too small, did not adequately adjust for confounders or assess the clinical utility of the measurements.

Chronic systemic inflammatory conditions, such as atherosclerosis, diabetes and obesity are associated with increased risk of stroke, which suggests that systemic inflammation may contribute to the development of stroke in humans. The hypothesis that systemic inflammation may induce brain pathology has been be tested in animals. Drake et al. [36] assessed inflammatory changes in the brain in rodent models of chronic, systemic inflammation. PET imaging revealed increased microglia activation in the brain of JCR-LA (corpulent) rats, which develop atherosclerosis and obesity, compared to the control lean strain. Immunostaining against Ibal confirmed reactive microgliosis in these animals. An atherogenic diet in apolipoprotein E knock-out (ApoE(-/-)) mice induced microglial activation in the brain parenchyma within 8 weeks and increased expression of vascular adhesion molecules. Focal lipid deposition and neuroinflammation in periventricular and cortical areas and profound recruitment of activated myeloid phagocytes, $T$ cells and granulocytes into the choroid plexus were also observed. In a small, preliminary study, patients at risk of stroke (multiple risk factors for stroke, with chronically elevated C-reactive protein, but negative MRI for brain pathology) exhibited increased inflammation in the brain, as indicated by PET imaging. These findings show that brain inflammation occurs in animals, and tentatively in humans, harbouring risk factors for stroke associated with elevated systemic inflammation. Thus a "primed" inflammatory environment in the brain may exist in individuals at risk of stroke and this can be adequately recapitulated in appropriate co-morbid animal models.

Stroke triggers an intense inflammatory response that could be a consequence of Toll-like receptors (TLRs) activation. However, the clinical significance and the therapeutic possibilities of TLR in stroke is not completely clear. In this study, we analyze the association between the expression of TLR2 and TLR4, inflammatory molecules and endogenous ligands, and clinical outcome of ischemic stroke patients, and we test the potential of TLR2/TLR4 and their endogenous ligands as therapeutic targets. For this purpose Brea et al. [35] included 110 patients with ischemic stroke finding that TLR2 and TLR4 are independently associated to poor outcome and correlated with higher serum levels of interleukin (IL) $1 \beta$, IL6, tumor necrosis factor $\alpha$, and VCAM1, and that TLR4 was independently associated to lesion volume. In addition, authors have developed an in vitro model to test the potential therapeutic value of blocking TLR2/TLR4 or their endogenous ligands. Cultured cells (monocytes and human umbilical vein endothelial cells) were treated with serum from ischemic stroke patients, showing a strong inflammatory response that was blocked when TLR2/4 or cellular fibronectin (cFN) or HSP60 were blocked. In conclusion, TLR2 and TLR4 are associated to outcome in stroke patients and 
TLR2/4 or their endogenous ligands, cFN/HSP60 could be new therapeutic targets for ischemic stroke.

Previous studies have shown that the concentration of peripheral inflammatory markers, particularly C-reactive protein (CRP), strongly correlates with stroke severity and independently predicts mortality and recurrent vascular events in patients with acute ischemic stroke. Youn et al. [35] conducted a study to clarify the relationship between inflammatory markers and stroke severity by means of volumetric measurement of infarct size. 96 patients who had laboratory investigations and magnetic resonance imaging scans were included retrospectively in this study. Diffusionweighted imaging (DWI) lesions were outlined using a semiautomatic threshold technique. Diffusion-weighted imaging lesion volumes were measured with MIPAV software (Medical Image Processing, Analysis and Visualization, version 4. 1. 1; National Institutes of Health, Bethesda, MD). The relationship between highly selective CRP (hs-CRP) levels and DWI infarct volume quartiles was examined. The mean age of patients was 66.9 years, and 50 patients $(51.2 \%)$ were male. There was a significant correlation between hs-CRP and DWI volumes (Spearman $\rho=0.239, \mathrm{P}=$. 010). The median hs-CRP values for successive volumes of DWI lesion quartiles (lowest to highest quartile) were as follows: 1. 17, 1. 14, 1. 63, and 3. $76(\mathrm{P}=.029)$. Higher hs-CRP levels were associated with larger infarct volumes in patients with acute ischemic stroke. These results suggest that elevated hs-CRP levels, reflecting a large volume of infarct, may serve as a helpful serologic marker in the evaluation of severity of acute ischemic stroke.

Animal research has characterized postischemic inflammation as a multi-facetted response involving activation of resident glia cells and recruitment of blood-derived leucocytes as well as cascades of humoral responses [35-36]. In the classical transient middle cerebral artery occlusion (tMCAO) model, hematogeneous cells including polymorphonuclear neutrophils (PMN) and macrophages rapidly infiltrate the ischemic region [37-40].

Neuroinflammation evolves as a multi-facetted response to focal cerebral ischemia. It involves activation of resident glia cell populations, recruitment of blood-derived leucocytes as well as humoral responses. Among these processes, phagocyte accumulation has been suggested to be a surrogate marker of neuroinflammation. Walberer et al. [41] in a rat model of permanent focal ischemia by embolisation of TiO2-spheres assessed key features of postischemic neuroinflammation by the means of histology, immunocytochemistry of glial activation and influx of hematogeneous cells, and quantitative PCR of TNF- $\alpha$, IL-1, IL-18, and iNOS mRNA. In the boundary zone of the infarct, a transition of ramified microglia into ameboid phagocytic microglia was accompanied by an upregulation of MHC class II on the cells after 3 days. By day 7, a hypercellular infiltrate consisting of activated microglia and phagocytic cells formed a thick rim around the ischemic infarct core. Interestingly, in the ischemic core microglia could only be observed at day 7 . TNF- $\alpha$ was induced rapidly within hours, IL-1 $\beta$ and iNOS peaked within days, and IL-18 later at around 1 week after ischemia. The macrosphere model closely resembles the characteristical dynamics of postischemic inflammation previously observed in human stroke. Authors therefore suggest that the macrosphere model is highly appropriate for studying the pathophysiology of stroke in a translational approach from rodent to human. Few data exist on the relationship between differential subpopulations of peripheral leukocytes and early cerebral infarct size in ischemic stroke. Using diffusion-weighted MR imaging (DWI), we assessed the relationship of early total and differential peripheral leukocyte counts and volume of ischemic tissue in acute stroke. A recent study [42] included patents that had laboratory investigations and neuroimaging collected within 24 hours of stroke onset. Total peripheral leukocyte counts and differential counts were analyzed individually and by quartiles. DWI lesions were outlined using a semiautomated threshold technique. The relationship between leu- kocyte quartiles and DWI infarct volumes was examined using multivariate quartile regression. 173 patients met study inclusion criteria. . Total leukocyte counts and DWI volumes showed a strong correlation (Spearman rho=0. 371, P<000. 1). Median DWI volumes $(\mathrm{mL})$ for successive neutrophil quartiles were: $1.3,1.3,3.2$, and 20. 4 ( $\mathrm{P}$ for trend $<0.001)$. Median DWI volumes $(\mathrm{mL})$ for successive lymphocyte quartiles were: $3.2,8.1,1.3$, and $1.5(\mathrm{P}=0$. 004). After multivariate analysis, larger DWI volume remained strongly associated with higher total leukocyte and neutrophil counts (both probability values $<0.001$ ), but not with lymphocyte count $(\mathrm{P}=0$. 4971). Compared with the lowest quartiles, DWI volumes were $8.7 \mathrm{~mL}$ and $12.9 \mathrm{~mL}$ larger in the highest quartiles of leukocyte and neutrophil counts, respectively. Higher peripheral leukocyte and neutrophil counts, but not lymphocyte counts, are associated with larger infarct volumes in acute ischemic stroke. Attenuating neutrophilic response early after ischemic stroke may be a viable therapeutic strategy and warrants further study Granulocytes are the first hematogenous cells that appear in the brain in response to focal ischemia.

After permanent occlusion of the MCA they accumulated in cerebral vessels within hours [43] before they invaded the infarct and its boundary zone. This process peaked at $24 \mathrm{hr}$ after infarction, thereafter the number of granulocytes rapidly declined. At day 7 granulocytes were only occasionally seen. Granulocyte infiltration was preceded by an increased expression of cytokine-induced neutrophil chemoattractant (CINC) [44]. There is evidencethat intravascular granulocyte accumulations reduce blood flow in the reperfusion phase and thereby contribute to extension of infarctions in transient cerebral ischemia, while the pathophysiological consequences of the parenchymal granulocyte invasion are unclear at present.

In addition to granulocytes, a significant number of $\mathrm{T}$ cells identified by immunocytochemistry invaded the infarct region [45]. After both permanent MCAO and photochemically induced ischemia, CD5+ T cells started to infiltrate the infarct region from day 1 onwards. At day 3 their number had increased and reached a peak around day 7 followed by a substantial decrease within the next 7 days. $T$ cells were preferentially located in the boundary zone of the infarctions often in close vicinity to blood vessels. The presence of $\mathrm{T}$ cells was surprising. $\mathrm{T}$ cell recruitment into the central nervous system (CNS) is usually observed in autoimmune and inflammatory diseases such as experimental autoimmune encephalomyelitis (EAE) [46].

Although cells of the central nervous system (CNS) constitutively express very low levels of MHC antigens, and express very low levels of MHC antigens, and are thus considered as 'immunologically silent', these cells, once stimulated, can express various cytokines and adhesion molecules. Three major cytokines, namely, tumor necrosis factor (TNF , interleukin (IL)-1, and IL-6 are produced by cultured brain cells after various stimuli. TNF- $\alpha$ is produced by astrocytes when the cells are stimulated by lipopolysaccharide (LPS), interferon (IFN)-7, and IL-1 $\beta$ [47]. Cultured astrocytes also produce high levels of IL-6, IL-8, granulocyte colony stimulating factor (G-CSF), M (macrophage)-CSF, and GM-CSF mRNAs and proteins when stimulated by IL-1 $\beta$ or TNF $\alpha$ [48]. Frei et al. [49] showed that GM-CSF and M-CSF were produced by astrocytes after stimulation with TNFa, IL-1, and LPS, suggesting that activated astrocytes may be responsible for the accumulation and activation of monocytes/microglia in the area of tissue injury.

Adipose tissue secretes numerous proinflammatory cytokines and is involved in the regulation of glucose metabolism. A study by Wang et al. [50] aimed to determine the effects of acute stroke on adipose inflammatory cytokine expression. In addition, because sympathetic activity is activated after acute stroke and catecholamines can regulate the expression of several adipocytokines, this study also evaluated whether alterations in adipose proinflammatory cytokines following acute stroke, if any, were medicated by 
sympathetic system. Acute ischemic brain injury was induced by ligating the right middle cerebral artery and bilateral common carotid arteries in male adult Sprague-Dawley rats. Adipose tumor necrosis factor- $\alpha$ (TNF- $\alpha$ ) and monocyte chemoattractant protein-1 (MCP-1) mRNA and protein levels were determined by RT-PCR and enzyme-linked immunoassay, respectively. The stroke rats developed glucose intolerance on days 1 and 2 after cerebral ischemic injury. The fasting blood insulin levels and insulin resistance index measured by homeostasis model assessment were higher in the stroke rats compared with the sham group. Epididymal adipose TNF- $\alpha$ and MCP-1 mRNA and protein levels were elevated one- to twofold, in association with increased macrophage infiltration into the adipose tissue. When the rats were treated with a nonselective $\beta$-adrenergic receptor blocker, propranolol, before induction of cerebral ischemic injury, the acute stroke-induced increase in TNF- $\alpha$ and MCP-1 was blocked, and fasting blood insulin concentration and homeostasis model assessment-insulin resistance were decreased. These results suggest a potential role of adipose pro-inflammatory cytokines induced by the sympathetic nervous system in the pathogenesis of glucose metabolic disorder in rats with acute ischemic stroke.

During the course of brain ischemia, inflammatory mechanisms both intrinsic to brain as well as blood are among the important mediators of focal cerebral injury [51]. With the onset of focal brain ischemia, microglia become activated within the penumbra and thereby facilitate further local neuronal injury via pathways that include poly(ADP-ribose) polymerase-1 activation and activation of multiple matrix metalloproteinases (MMPs). The activation of MMPs disrupts the blood-brain barrier, alters microvascular endothelial function and impairs the functional integrity of the neurovascular unit. These events promote the entry of peripheral leukocytes into brain and expose "protected" cerebral elements to peripheral immune surveillance systems.

Cerebral ischaemic injury is associated with the induction of a series of inflammatory events, including the infiltration of circulating immune cells (neutrophils and monocytes) and activation of resident cells, including microglia, astrocytes and endothelial cells [51]. These cells can express, release and respond to proinflammatory mediators such as cytokines [5]. As inflammation is a ubiquitous response to tissue injury, this is to be expected; however, it is clear from experimental data.

Animal models of focal ischaemia induced by middle cerebral artery occlusion (MCAO) provide most evidence for cellular inflammatory responses in stroke. Permanent MCAO results in a modest neutrophil infiltration at $24 \mathrm{~h}$ after ischaemia, predominantly around arterial vessels at the margins of infarction, whereas MCAO with subsequent reperfusion is associated with substantial infiltration by neutrophils throughout the entire infarct [52]. Neutrophil accumulation in small vessels including capillaries in the peri-infarct region may contribute to the 'no reflow' phenomenon a failure of tissue to reperfuse despite recanalization of the main vessel occluded [53].

Inflammatory cells such as neutrophils and macrophages infiltrate into the ischemic brain in various animal models of ischemic stroke [53, 54, 55]. According to an animal study using the rat [54] ubjected to permanent middle cerebral artery occlusion (MCAO), polymorphonuclear leukocytes first appeared in the capillary lumen at $30 \mathrm{~min}$, peaked at $12 \mathrm{~h}$, whereas they first appeared in the parenchyma at $12 \mathrm{~h}$, peaked at $24 \mathrm{~h}$, and diminished in number thereafter. In addition, inherent cells such as astrocytes, microglia, or endothelia have been found to be activated by cerebral injuries including ischemic stroke. These cells then become immunologically reactive and interact with each other by producing substances including cytokines and adhesion molecules. These molecules appear to be responsible for the accumulation of inflammatory cells in the injured brain, and the resulting immunologic-inflammatory cascade produces an environment that may affect the survival of neurons subjected to ischemic injury constitutively express very low levels of MHC antigens, and are thus considered as 'immunologically silent', these cells, once stimulated, can express various cytokines and adhesion molecules.

Three major cytokines, namely, tumor necrosis factor (TNF, interleukin (IL)-1, and IL- 6 are produced by cultured brain cells after various stimuli.

The differences between models lead to problems in characterizing the therapeutic response to inhibitors of neutrophil migration and marked clinical heterogeneity in timing of reperfusion may have contributed to the failure of such agents to show benefit in clinical trials.

On this basis in acute stroke, there are therefore several areas in which inflammation may be relevant: as an indication of underlying vascular disease predisposing to stroke; as a precipitating process acting as a trigger for an acute event; once a stroke has occurred, as an acute response to tissue injury, possibly with a role in exacerbating the injury or its consequences; as a prognostic marker in the acute phase of stroke; or as a prognostic marker for recurrent cardiovascular events - both stroke and extracerebral events. Any or all of these might give rise to therapeutic targets.

In this review, we provide basic science evidence concerning role of inflammation in neuronal damage after acute ischemic stroke and we will discuss some potential inflammatory therapeutic targets in acute stroke treatment.

Elevated levels of C-reactive protein (CRP), a proinflammatory marker, are associated with reduced systemic endotheliumdependent NO-mediated dilation in patients with coronary artery disease; however, the direct effect of CRP on coronary microvascular reactivity remains unknown. Herein, Qamirani et al. [56] examined whether CRP can modulate endothelium-dependent NOmediated dilation of coronary arterioles and whether proinflammatory signaling pathways such as stress-activated protein kinases (p38 and c-Jun N-terminal kinase [JNK]) and oxidative stress are involved in the CRP-mediated effect. Porcine coronary arterioles were isolated and pressurized without flow for in vitro study. Intraluminal treatment with a clinically relevant concentration of CRP (7 microg/mL; 1 hour) significantly attenuated the NO release and vasodilation to serotonin. Further incubation with the NO precursor 1-arginine $(3 \mathrm{mmol} / \mathrm{L})$ partially restored serotonin-induced vasodilation. In the presence of superoxide scavenger 4-hydroxy-2, 2, 6, 6tetramethylpiperidine-1-oxyl (TEMPOL), NAD(P)H oxidase inhibitor apocynin, or p38 kinase (an upstream activator of $\mathrm{NAD}(\mathrm{P}) \mathrm{H}$ oxidase) inhibitor SB203850, but not xanthine oxidase inhibitor allopurinol or JNK inhibitor SP600125, the detrimental effect of $\mathrm{CRP}$ on serotonin-induced dilation was prevented. Dihydroethidium staining showed that CRP produced SB203850- and TEMPOLsensitive superoxide production in the arteriolar endothelium. CRP treatment of coronary arterioles significantly increased NAD $(\mathrm{P}) \mathrm{H}$ oxidase activity. Authors concluded that CRP inhibits endotheliumdependent NO-mediated dilation in coronary arterioles by producing superoxide from $\mathrm{NAD}(\mathrm{P}) \mathrm{H}$ oxidase via $\mathrm{p} 38$ kinase activation. By impairing endothelium-dependent NO-mediated vasoreactivity, CRP could facilitate the initiation of numerous cardiovascular diseases.

Furthermore CRP seems to have a direct pathogenetic role in brain ischemia . Increased mortality after stroke is associated with brain edema formation and high plasma levels of the acute phase reactant C-reactive protein (CRP). A recent study [57] was designed to examine whether CRP directly affects blood-brain barrier stability and to analyze the underlying signaling pathways. Authors used a cell coculture model of the blood-brain barrier and the guinea pig isolated whole brain preparation and they showed that CRP at clinically relevant concentrations (10 to $20 \mathrm{microg} / \mathrm{mL}$ ) causes a disruption of the blood-brain barrier in both approaches. The results of this study further demonstrate CRP-induced activa- 
tion of surface Fc $\gamma$ receptors CD16/32 followed by p38-mitogenactivated protein kinase-dependent reactive oxygen species formation by the $\mathrm{NAD}(\mathrm{P}) \mathrm{H}$-oxidase. The resulting oxidative stress increased myosin light chain kinase activity leading to an activation of the contractile machinery. Blocking myosin light chain phosphorylation prevented the CRP-induced blood-brain barrier breakdown and the disruption of tight junctions. These data identify a previously unrecognized mechanism linking CRP and brain edema formation and present a signaling pathway that offers new sites of therapeutic intervention

Several studies [58-60] show that C-reactive protein (CRP), an inflammatory marker, is associated with stroke outcomes and future vascular events. It is not clear whether this is due a direct doseresponse effect or rather an epiphenomenon. Idicula et al. [61] studied the effect of CRP measured within 24 hours after stroke onset on functional outcome, mortality and future vascular events. Authors prospectively studied 498 patients with ischemic stroke who were admitted within 24 hours after the onset of symptoms. CRP and NIH stroke scale (NIHSS) were measured at the time of admission. Short-term functional outcome was measured by modified Rankin scale (mRS) and Barthel ADL index (BI) 7 days after admission. Patients were followed for up to 2. 5 years for long-term mortality and future vascular events data. The median CRP at admission was $3 \mathrm{mg} / \mathrm{L}$. High CRP was associated with high NIHSS (p $=0.01)$ and high long-term mortality $(\mathrm{p}<0.0001)$. After adjusting for confounding variables, high CRP remained to be associated with high NIHSS $(\mathrm{p}=0.02)$ and high long-term mortality $(\mathrm{p}=0$. 002). High CRP was associated with poor short-term functional outcomes $(\mathrm{mRS}>3$; $\mathrm{BI}<95)(\mathrm{p}=0.01 ; \mathrm{p}=0.03)$. However, the association was not significant after adjusting for confounding variables including stroke severity $(\mathrm{p}=0.98 ; \mathrm{p}=0.88)$. High CRP was not associated with future vascular events $(\mathrm{p}=0.98)$. Authors concluded that admission CRP is associated with stroke severity and long-term mortality when measured at least 24 hours after onset. There is a crude association between high CRP and short-term functional outcome which is likely secondary to stroke severity. $\mathrm{CRP}$ is an independent predictor of long-term mortality after ischemic stroke.

Recent investigations [62-66] demonstrated that mRNAs of above cytokines were induced in the ischemic rat brain. TNF- is a pleiotropic cytokine that mediates key roles in many physiological and pathological cellular processes including acute and chronic inflammation, programmed cell death or apoptosis, anti-tumor responses, and infection [67].

The source of these circulating cytokines in patients with ischemic stroke remains unknown. However, Tarkowski et al. [68] serially measured serum and cerebrospinal fluid IL-1 /3 and IL-6 in patients with ischemic stroke and found that the levels of these cytokines were much higher in the cerebrospinal fluid than in the serum. These results are highly suggestive of intrathecal production of the cytokines. The level of early production of IL-6 in cerebrospinal fluid was found to be significantly correlated with the size of brain lesion. In addition, Mathiesen et al. [69] found markedly (more than 300 fold) increased level of IL- 6 in cerebrospinal fluid following subarachnoid hemorrhage, which was not paralleled by the amount of the cytokine in the serum. The elevation of cytokine was more marked at day 6 than day 3 or 9 , and could be associated with the development of delayed ischemic deficits. Increased levels of IL-1 and IL-6 in ventricular fluid was also detected in patents with severe head injuries [70] .

A growing body of evidence from studies using both preclinical animal models and human samples collected from ischemic stroke patients suggests TNF is also involved in the development of ischemic brain lesions. TNF is rapidly expressed in ischemic brain after the onset of the disease [71]. The upregulation of TNF also leads to the damage of the blood brain barrier (BBB) [72], as well as neuronal cell death during the stroke [73]. On the one hand, TNF was shown to be responsible for the neuronal death in an oxygenglucose deprivation (OGD) model in vitro and in an ischemic brain damage model in vivo [74]. TNF- is also involved in apoptotic death of neurons exhibiting peripherin aggregates [73]. BALB/C mice pretreated with $\mathrm{F}$ - . intracisternally $48 \mathrm{~h}$ prior to MCAO also showed significant reduction in infarct size [75], presumably through the induction of the ischemic preconditioning (IPC) which can be protective against subsequent brain injury.

Interleukin-1 (IL-1) family consists of IL-1 , IL-1 . converting enzyme (ICE)/caspase-1, IL-1 , IL-1 receptors I (IL-1RI) and II (IL-1RII), and IL-1 receptor antagonist (IL-1 ra) IL-1 is cleaved from the precursor protein, pro-IL- 1 , by ICE. IL-1RI is a signaling receptor, whereas IL-1RII is a truncated receptor that serves as a decoy to inhibit IL-1 activity . IL-1ra is an endogenous protein that inhibits. IL-1 . activity by competitive binding to the IL-1RI [76]. Increased expression of IL-1 . and IL-1RI in hypoxic brain cells [44] and peri-infarct tissues [77, 78] has been linked to ischemic brain injury. Clinical studies have shown that intrathecal [79] and systemic $[80,81]$ increases in IL-1 , interleukin-8 (IL-8) and interleukin-17 (IL-17) correlate with neurological impairment in stroke patients.

On this basis the presence of an inflammatory response in the pathophysiology of acute brain ischemia is relatively well established. Cytokines such as interleukin-6 (IL-6), tumor necrosis factor- $\alpha$ (TNF- $\alpha$ ) appear to be crucial mediators of such responses, however much remains unknown about their complex interactions in the setting of clinical stroke $[82,83]$.

Increased production of pro-inflammatory cytokines has been observed in experimental models of brain ischemia as well as in patients with acute stroke $[84,85]$ and may be associated with large cerebral infarct volume and poor stroke outcome [86, 87].

Inflammatory cytokine families and their receptors expressed in brain tissue may be exploited as drug targets to control brain inflammation resulting from the ischemic insult. Several strategies have been tested to block inflammatory effects of cytokines and chemokines, including neutralizing antibodies, soluble cytokine/chemokine receptors, inhibitors of cytokine/chemokine synthesis, and anti-inflammatory cytokines.

The balance between pro-inflammatory and anti-inflammatory cytokine molecules is altered in the early phase of acute ischemic stroke. Some investigators have reported the role of IL-10 as the main downregulator of deleterious effect of proinflammatory cytokines [88, 89].

Kes et al. [90] to explore the difference in concentration of IL6 , TNF- $\alpha$ and IL-10 between acute ischemic stroke patients and control individuals analyzed the association of plasma cytokine concentration with stroke severity at admission assessed by NIHSS as prognostic score and stroke outcome in 90 days assessed by Barthel index (BI) and modified Rankin scale (mRS). These authors showed that Il-6 was increased in patients relative to controls and this increase was associated with severe stroke and worse outcome (assessed by BI and mRS, respectively), whereas IL-10 was decreased and associated with better outcome.

On this basis An immunoinflammatory cascade. involving endothelial and coagulation functions represents the pathogenetic background of neuronal damage in ischemic stroke. Indeed, there is evidence that brain injury, secondary to arterial occlusion, is characterized by acute local inflammation

\section{INFLAMMATION IN ACUTE STROKE SUBTYPES}

Clinical studies have reported increased levels of proinflammatory cytokines and adhesion molecules in the peripheral blood and cerebrospinal fluid (CSF) of patients with ischemic stroke. 
High IL-6 concentrations in CSF and plasma have been associated with larger infarct size, neurological deterioration, and poor outcome independently of the stroke subtype.

However, although accumulating evidence suggests that inflammatory-mediated damage plays a role in brain ischemia, for long time it remains unclear whether inflammation also intervenes in a same intensity in each diagnostic subtype of ischemic stroke.

Castellanos et al. [91] sought to investigate the role of proinflammatory molecules in the early worsening and outcome of acute lacunar stroke. Authors performed a secondary analysis of 113 consecutive patients with lacunar infarction included within the first 24 hours of the onset of symptoms in a previous study aimed at investigating clinical and biochemical factors of early neurological deterioration (END). END was defined as a fall of $>$ or $=1$ points in the motor items of Canadian Stroke Scale between inclusion and 48 hours. Poor outcome at 3 months was considered death or Barthel Index <85. Interleukin-6 (IL-6), tumor necrosis factor-alpha (TNFalpha), and intercellular adhesion molecule-1 (ICAM-1) were determined by enzyme-linked immunoabsorbent assay in blood samples obtained on admission. END was recorded in 27 patients $(23$. $9 \%$ ); poor outcome was noted in $26(23 \%)$. Median (quartiles) concentrations in plasma of TNF-alpha [16.5 pg/mL (13. 7 and 21.2 $\mathrm{pg} / \mathrm{mL}$ ) versus $7.5 \mathrm{pg} / \mathrm{mL}$ (6. 2 and $9.0 \mathrm{pg} / \mathrm{mL})$ ], IL-6 [28. 8 $\mathrm{pg} / \mathrm{mL}(22.5$ and $35.7 \mathrm{pg} / \mathrm{mL}$ ) versus $11.5 \mathrm{pg} / \mathrm{mL}(8.5$ and 16.2 $\mathrm{pg} / \mathrm{mL}$ )], and ICAM-1 [285 pg/mL (219 and $315 \mathrm{pg} / \mathrm{mL})$ versus 158 $\mathrm{pg} / \mathrm{mL}$ (137 and $187 \mathrm{pg} / \mathrm{mL}$ )] were significantly higher in patients who had END than in those with nonprogressing strokes $(\mathrm{P}<0$. 001). Significant differences were also observed between patients with poor and good outcome at 3 months. Logistic regression analysis after adjustment for potential confounders showed that TNF-alpha $>14 \mathrm{pg} / \mathrm{mL}$ and ICAM- $1>208 \mathrm{pg} / \mathrm{mL}$ were independently associated with both END (OR, 511; 95\% CI, 17 to 4937; $\mathrm{P}<0$. 001; and OR, 315; 95\% CI, 17 to 5748 ; $\mathrm{P}<0$. 001, respectively) and poor outcome at 3 months (OR, 3. 0; 95\% CI, 1.0 to 8. 5; $\mathrm{P}=0.042$; and $\mathrm{OR}, 4$. 2; $95 \% \mathrm{CI}, 1.3$ to 13.6 ; $\mathrm{P}<0$. 015, respectively).

This study demonstrates in a large series of patients with lacunar infarctions an independent association of high levels of inflammatory molecules in blood with END and poor outcome. The effect of these compounds on END was stronger because the outcome depends particularly on baseline stroke severity. The mechanisms involved in END of lacunar infarctions have not been clearly established, and it seems unlikely that those factors currently accepted as contributing to worsening in other stroke subtypes offer a full explanation in the case of lacunar stroke.

TNF- $\alpha$ promotes the expression of adhesion molecules such as ICAM-1 on the endothelium, facilitating leukocyte adherence and migration from capillaries into the brain, microvessel occlusion, and subsequently a progressive reduction in blood flow [92, 93]. The accumulation of polymorphonuclear neutrophil leukocytes in the ischemic area as a result of the inflammatory process has been proved in a few clinical observations with brain scintigraphy or brain SPECT with labeled leukocytes. 37, 38 High levels of TNF- $\alpha$ have been detected as soon as 15 hours in brain samples of stroke victims, peaking during days 2 and 3,39 and plasma determinations after acute stroke have demonstrated an early activation of ICAM1 , which peaks within 24 hours of cerebral ischemia [94, 95]. These findings are in accordance with our results because we found increased TNF- $\alpha$ and ICAM-1 levels within 24 hours (mean, 11 hours) of the onset of lacunar stroke. High levels of adhesion molecules may reflect a prior condition of chronic endothelial activation secondary to risk factors for atherosclerosis such as hypertension, which was significantly more frequent in our patients who had END. In this study, however, high ICAM-1 levels remained independently associated with subsequent END after controlling for the history of hypertension in the logistic analysis.
An interesting finding in this study is that inflammatory molecules contributed to END after adjustment for glutamate and GABA concentrations in blood and that the ORs for TNF- $\alpha$ and ICAM- 1 were even higher than that for glutamate concentrations. These results suggest that inflammation may have an additional and stronger role than excitotoxicity in END of lacunar infarctions. On the other hand, inflammatory and excitatory mechanisms might cooperate in the progression of lacunar stroke because we have found a significant correlation between glutamate or GABA concentration and inflammatory markers in blood. Furthermore, as occurred with amino acid concentrations, [96] authors have observed higher levels of inflammatory molecules in patients with lacunar infarctions located in basal ganglia and brainstem than in those with white matter infarctions, so excitotoxicity and inflammation might represent sequential and interacting processes in the progression of lacunar stroke, particularly in brain areas with a high density of glutamatergic neurons [97]. This hypothesis is supported by experimental data suggesting that cytokines influence glutamate receptor mediated excitotoxicity.

A study by Sotgiu et al. [98] was designed to evaluate the serum concentrations of a panel of biomarkers known to be variably associated with ischemic stroke (IL-1b, TNF-a, IL-6, IL-8, MCP-1, VCAM-1, ICAM-1, MMP-2/9 and the brain-derived neurotrophic factor, BDNF) in a consecutive series of patients with acute brain ischemia without concomitant infections, and to correlate their level with the extent of brain lesion as documented by computed tomography $(\mathrm{CT})$ and neurological outcome both at study entry and at 3 month period.

Infarct size was calculated on computed tomography (CT) scans by means of the Cavalieri's method. Neurological impairment was scored by using the Glasgow Coma Scale, Glasgow Outcome Scale and National Institutes of Health (NIH) scales at stroke onset and 3month follow-up. Some markers showed a direct significant correlation with both initial and final NIH scale and with infarct size, particularly tumor necrosis factor alpha (TNF-alpha) $(\mathrm{P}=0.002)$, intercellular adhesion molecule-1 $(\mathrm{P}<0.01)$ and matrix metalloproteinase-2/9 $(\mathrm{P}=0$. 001). In contrast to previous reports, interleukin-6 (IL-6) serum level showed a significant inverse correlation with both final neurological impairment and infarct size $(\mathrm{P}<0.001)$. This novel finding allows the authors suggesting that IL-6, in the context of a complex pro-inflammatory network occurring during stroke, is associated with neuroprotection rather than neurotoxicity in patients with ischemic brain injury.

It has become increasingly evident that the inflammatory response plays an important role in the pathogenesis of neuronal damage following stroke. Pro-inflammatory cytokines, including tumor necrosis factor (TNF)- $\alpha$, interleukin (IL)-1 $\beta$ and IL-6 are secreted in the ischemic region by activated immune cells, which drive the inflammatory process and accelerate additional inflammatory processes by inducing inflammatory molecules, such as intercellular adhesion molecules (ICAM), vascular cell adhesion molecules-1 (VCAM-1) and selectins [99]. These inflammatory modulators recruit more circulating leukocytes that infiltrate into the ischemic region and lead to further loss of neuronal cells and brain tissue, resulting in an increase of cerebral infarct size [99, 100, 101]

Recently our group [102] conducted a study with the aim to evaluate possible differences in the immunoinflammatory activation of acute phase and in thrombotic/fibrinolitic pathway (TPA and PAI-1 plasma levels) in stroke patients in relation to time of symptoms onset, diabetic state and diagnostic subtype of stroke. We enrolled 60 consecutive patients with a diagnosis of acute ischemic stroke (32 with type 2 diabetes and 28 non-diabetics), admitted to the Internal Medicine Department at the University of Palermo between November 2002 and January 2005, and 123 consecutive hospitalized control patients without a diagnosis of acute ischemic stroke. Stroke was defined by focal neurological signs or symptoms thought to be of vascular origin that persisted for $>24$ hours, con- 
firmed by brain CT and /or MRI in baseline conditions and brain CT with contrast medium after 48-72 hours. Patients were defined as type 2 diabetics if they had known diabetes treated with diet, oral hypoglycemic drugs or insulin before stroke. We excluded patients with stress hyperglycaemia and new onset diabetes because we intended to analyze only chronic hyperglycaemia effects on immunoinflammatory activation. Patients with inflammatory or infectious diseases, acute myocardial infarction (AMI), venous thromboembolism (DVT or EP), cancer, hematological diseases and severe renal or liver failure, as well as those who were under treatment with anti-inflammatory drugs, were excluded. The type of acute ischemic stroke was classified according to the TOAST classification (6): 1) Large artery atherosclerosis (LAAS); 2) cardioembolic infarct (CEI); 3) LACunar infarct (LAC); 4) stroke of other determined etiology (ODE); 5) stroke of undetermined etiology (UDE). All the ischemic stroke patients had medical history recording potential stroke risk factors and underwent; blood and coagulation tests; 12-lead ECG; 24 hour electrocardiography monitoring; transthoracic echocardiography; carotid ultrasound; brain CT or MRI at admission (repeated between the third and the seventh days of stroke onset).

Blood samples from each subject enrolled were drawn within 72 hours from and 7-10 days after symptoms onset and they were centrifuged $(10.000 \mathrm{~g})$, and the resulting supernatant was immediately frozen at $-80^{\circ} \mathrm{C}$ until analysis was completed. We evaluated plasma levels of IL-1 $\beta$, TNF- $\alpha$, IL- 6 and IL-10, E-selectin, Pselectin, sICAM-1 and sVCAM-1 as markers of immunoinflammatory activation, VWF plasma levels as a marker of endothelial dysfunction, TPA antigen and PAI-1 plasma levels as a marker of a prothrombotic state. Neurological deficit score at Scandinavian Stroke Scale (SSS) on admission and disability degree at discharge by Rankin disability scale were obtained for stroke patients. SSS assesses neurological deficit through an evaluation of consciousness level, eye movement, strength in the arms, hand and legs, orientation, language, facial weakness and gait, giving rise to a score ranging from 58 (absence of deficit) to 0 (death). According to the modified Rankin disability score, functional ability after stroke was graded by a score ranging from 1 (no significant disability) to 6 (death).

Stroke patients exhibited significantly higher plasma levels of PAI-1. Of the stroke patients, 31 were classified as lacunar and 29 as non-lacunar. Concerning inflammatory variables, the stroke patients versus controls exhibited, at 24-72h after stroke onset, higher levels of IL-1 $\beta$, TNF- $\alpha$, E-Selectin, VCAM-1, ICAM-1, VWF and white body cells (WBC ). Among our stroke patients, diabetic subjects vs non-diabetics were significantly younger [68 yrs (63-72) vs 78 yrs (68-88); $\mathrm{p}=0$. 004]. Diabetics also showed no significant difference regarding the plasma levels of immunoinflammatory variables, but exhibited significantly higher plasma levels of PAI-1 [163 pg/ml (127. 5-192. 5) vs $125 \mathrm{pg} / \mathrm{ml}$ (104. 75-159); $\mathrm{p}=0.03$ ]. To evaluate immunoinflammatory activation in relation to diagnostic subtype, we examined the relationship between the analyzed variables and TOAST subtype, respectively lacunar and nonlacunar The patients with lacunar stroke in comparison with nonlacunar ones were significantly younger [66. 5 yrs (60-76. 5) vs 75 yrs (66-86); $\mathrm{p}=0$. 007), had significantly higher glucose blood levels (217. $5 \mathrm{mg} / \mathrm{dl}$ (101-244) vs $107 \mathrm{mg} / \mathrm{dl}$ (96-226); $\mathrm{p}=0.025]$ and a significantly higher SSS score $[45$ (47-50. 7) vs 36 (9-39); $\mathrm{p}=0$. 0001). Moreover, with regard to immuno-inflammatory variables, the patients with lacunar strokes in comparison with subjects with non-lacunar stroke exhibited, 24-72h after stroke onset, lower plasma levels of IL-1 $\beta$ and TNF- $\alpha$. At 7-10 days after stroke onset TNF- $\alpha$ [83. $5 \mathrm{pg} / \mathrm{ml}(65.25-200)$ vs $258 \mathrm{pg} / \mathrm{ml}(140-1000) ; \mathrm{p}=0$. $001]$ and IL-1 $\beta$ [5 pg/ml (3-8. 75) vs $8 \mathrm{pg} / \mathrm{ml}(5-11) ; \mathrm{p}=0$. 0001] plasma levels remained lower in patients with lacunar stroke, but subjects with non-lacunar stroke also exhibited higher plasma levels of P-selectine [4. $5 \mathrm{ng} / \mathrm{ml}$ (1. 8-6. 5) vs $1.8 \mathrm{ng} / \mathrm{ml}$ (2. 17-5. 95); $\mathrm{p}=0.04]$ and ICAM-1[19. $1 \mathrm{ng} / \mathrm{ml}(17-23)$ vs $15.6 \mathrm{ng} / \mathrm{ml}(14.25$ 22. 75); $\mathrm{p}=0$. 01]. Moreover, to assess possible differences in relation to diabetes, patients with lacunar and non-lacunar stroke were further evaluated in relation to diabetic or non-diabetic state. Diabetic patients with lacunar stroke in comparison with non-diabetic patients with lacunar stroke and in comparison with diabetics and non-diabetics with non-lacunar stroke, were significantly younger [64 yrs (60-71) vs 78 yrs (64-78. 5) vs 69 yrs (64. 2-81. 7) vs 81 yrs (68-89); $\mathrm{p}=0.001)$, had significantly higher systolic blood pressure levels [165 mmHg (135-175) vs $155 \mathrm{mmHg}(153.7-171.2)$ vs 150 (141. 2-155. 7; $\mathrm{p}=0$. 0001], significantly lower diastolic blood pressure (DBP) levels (82. $5 \mathrm{mmHg}$ (80-96. 2) vs $95 \mathrm{mmHg}$ (92. 5-95) vs $92.5 \mathrm{mmHg}(75-100)$ vs $90 \mathrm{mmHg}(80-100) ; \mathrm{p}=0$. 0001] and a significantly higher SSS score [50 (45. 5-50.2) vs 47.5 (47-51) vs $37(31-40.7)$ vs 34 (2-39); $p=0.0001]$.

Concerning inflammatory variables, the diabetic patients with lacunar stroke in comparison with non-diabetic patients with lacunar stroke and in comparison with diabetics and non-diabetics with non-lacunar stroke exhibited, 24-72h after stroke onset, significantly lower levels of IL- $1 \beta$, TNF- $\alpha$ and ICAM-1. In diabetic patients with lacunar stroke, 7-10 days after stroke onset, plasma levels of TNF- $\alpha$ [64. $5 \mathrm{pg} / \mathrm{ml}$ (40. 5-99. 2) vs $145 \mathrm{pg} / \mathrm{ml}(92-415)$ vs $467 \mathrm{pg} / \mathrm{ml}(171.2-550)$ vs $314.5 \mathrm{pg} / \mathrm{ml}(88.7-447) ; \mathrm{p}=0.001]$, IL$1 \beta[3.5 \mathrm{pg} / \mathrm{ml}(1-5)$ vs $6 \mathrm{pg} / \mathrm{ml}(5-6.25)$ vs $6 \mathrm{pg} / \mathrm{ml}(4.2-11.5)$ vs 8 $\mathrm{pg} / \mathrm{ml}(6-10) ; \mathrm{p}=0.013]$ and ICAM-1 [14. $9 \mathrm{ng} / \mathrm{ml}(11.8-19.2) \mathrm{vs}$ 16. $2 \mathrm{ng} / \mathrm{ml}(14.3-17.7)$ vs $19.2(15-21)$ vs $18.4(15.2-22) ; \mathrm{p}=0$. 006] remained significantly lower in comparison with non-diabetic patients with lacunar stroke and with diabetics and non-diabetics with non-lacunar stroke .

Our study, consistent with published data, [103, 104] we observed higher plasma levels of IL- $1 \beta$, TNF- $\alpha$, IL- 6 , E and P selectin, ICAM-1 and VCAM-1 and VWF in stroke patients in comparison to control subjects without acute ischemic stroke. It has been assumed that IL- $1 \beta$ is the primary form of IL- 1 involved in ischemic brain damage [104-107] TNF- $\alpha$ plays a pivotal role in inflammatory processes that increase leukocyte-endothelial cell adhesion molecule expression, increase leukocyte adherence to blood vessels and their subsequent infiltration into the brain, and activates the endothelium for pro-coagulation activity [105]. Interleukin-6 (IL-6) is an inflammatory cytokine which can damage the endothelium and lead to the initiation of atherosclerosis, and it is also related to ischemic neural damage and clinical worsening [107].

On the other hand, we also report no significant difference in IL-1 $\beta$, TNF- $\alpha$, IL-6 E and P selectin, ICAM-1 and VCAM- 1 and VWF plasma levels between diabetic and non-diabetic stroke patients, but diabetics, in comparison to non-diabetic ones, exhibited higher plasma levels of PAI-1. This result is particularly interesting and could be related to three factors:

1. The role of PAI-1 as acute phase molecule [108].

2. The reported induction of plasminogen activator inhibitor type-1 by hyperglycemia, hyperinsulinaemia and precursor of insulin [109] that in diabetic subjects, and particularly in diabetic stroke patients, are particularly high.

3. The prothrombotic state of the diabetic subjects [110].

Therefore, this possibly accounts for the immunoinflammatory activation of the acute phase of stroke, but probably reflects the metabolic pattern of diabetes, and it could partially explain the reported higher recurrence of ischemic stroke in diabetic subjects .

Our study could highlight possible peculiarities of acute cerebral ischemia in diabetics in comparison with non-diabetics and our results appear particularly interesting regarding the differences of immunoinflammatory activation in relation to TOAST subtype. In fact, patients with lacunar strokes in comparison with subjects with non-lacunar strokes, exhibited, 24-72h after stroke onset, significantly lower levels of TNF- $\alpha$ and IL1- $\beta$. TOAST classification 
underlined that ischemic stroke is not a unique pathological condition but a group of neurological syndromes with different etiologic, clinical and prognostic features, so it is possible that each diagnostic subtype could be characterized by a different pathogenetic substrate with various grades of immunoinflammatory activation. Possibly this lower intensity of cytokines activation of the acute phase could be related to the lower extent of lacunar strokes, with a possible relationship between lower TNF- $\alpha$ and IL-1 $\beta$ plasma levels of the acute phase and the minor acute neurological deficit of lacunar subtype, previously reported by our group $[111,112]$ and also reported in this study. Furthermore, 7-10 days after stroke onset, this trend of inflammatory activation is confirmed with additional evidence of significantly higher levels of P-Selectin in patients with non-lacunar strokes in comparison to subjects with lacunar ones, owing to a possibly less rapid slope in levels of this inflammation mediator in comparison to lacunar patients. P-Selectin, as a result of platelet activation and endothelial damage, plays an important role in the pathogenesis of ischemic infarct [112]. Indeed P-Selectin expression leads to adhesion of leukocytes and subsequent release of cytokines and oxygen radicals, activation of coagulation and stimulation of endothelial cells and platelets, in an ongoing cycle of neural tissue damage. Our findings regarding a higher increase of $\mathrm{P}$ selectin plasma levels 7-10 days after stroke onset, in patients with non-lacunar stroke vs subjects with lacunar ones, could underline the contribution of atherosclerotic vascular disease to the increase of soluble adhesion molecules, and probably suggests the important role of leukocytes and platelets in the late-acute phases of brain infarction, particularly in atherothrombotic strokes.

In our study, lacunar stroke of diabetic patients appears to be peculiar in comparison with non-diabetic lacunar stroke. The former are characterized by significantly lower levels of IL $1 \beta$ and TNF- $\alpha$, both at $24-72 \mathrm{~h}$ and 7-10 days after stroke. This interesting finding could explain our previous data $[110,111]$ concerning a more favorable outcome in diabetic patients owing to the higher prevalence of lacunar strokes and it could provide a possible explanation for this issue, based on the minor grade of immunoinflammatory activation of both early and late acute phase of ischemic stroke in these patients, possibly related to the minor extent of ischemic brain damage. It is likely that a minor grade of chronic inflammatory response in cerebral small vessels could be involved in diabetic brain microangiopathy owing to the minor diameter of the pathogenesis of the diabetic lacunae [113]. Moreover, we observed a lower grade of ICAM 1 activation in diabetic patients with lacunar stroke. Intracellular adhesion molecule-1 (ICAM-1) is a member of the immunoglobulin superfamily and the principal ligand for the leukocyte function-associated antigen-1 (LFA-1), a member of the integrin superfamily [114]. One function of ICAM-1/LFA-1 adhesion system is to assist leukocyte movement into tissue and LFA-1 is constitutively expressed by brain microglial cells [114]. Our finding is possibly related to a lower extension of lacunar infarcts with a lower grade of recruitment of leukocytes into infarct area and consecutively with a minor release of bioactive substances, e. g. reactive oxygen metabolites, granular enzymes or cytokines, that induce damage to neural cells.

In conclusion, it is very clear that ischemic stroke is a multifactorial and dynamic process. We have confirmed the immunoinflammatory activation of the acute phase in patients with acute ischemic stroke and we measured the temporal event from strokeonset-dependent relationship between inflammation of the acute phase, diabetic state and diagnostic subtype with particularly lower median plasma levels of TNF- $\alpha$, IL1 $\beta$, P-selectin and ICAM- 1 in lacunar diabetic patients. Therefore, the degree of inflammatory response to ischemic stroke may be variable. A possible explanation for this variability, in addition to the infarct size, stroke subtype, time from symptoms onset and metabolic background as we report in our study, could be the genetic control of immunoinflammatory response related to polymorphism of the candidate genes, as recently shown by Pola et al. [115] who reported how proinflammatory genetic genotypes (IL-6 GG, ICAM-1 EE, E-Selectin AA) are significantly more common in subjects with stroke history. Only prospective, well-designed studies will provide the currently lacking information and will gradually improve our knowledge in the field of genetic polymorphisms in acute ischemic stroke practice

At extracranial arterial territories, inflammation plays a crucial role mediating all the stages of the atherosclerosis process [116] Similarly, thrombosis and defective fibrinolysis may also contribute to the progression of atherosclerotic lesions [117]. Interestingly, both mechanisms might have a relevant role in the pathogenesis of intracranial large artery atherosclerosis and ischemic stroke.

On this basis, it would be interesting to evaluate inflammatory and plasma levels of thrombotic/fibrinolytic markers in relation to each diagnostic subtype of ischemic stroke.

One study examined the inflammatory pathway in the lacunar subtype [118] whereas no study, to our knowledge, evaluated the inflammatory pathway in other subtypes of ischemic stroke. Fewstudies have examined the relationship between blood levels of inflammatory biomarkers and stroke outcome [119].

On this basis we conducted another study with the aim to evaluate, in patients with acute ischemic stroke, plasma levels of immuno-inflammatory markers, and endogenous activators and inhibitors of fibrinolysis plasma levels in relation to the diagnostic subtype of stroke, and to evaluate the relationship between these variables with clinical outcome and infarct site.

In our study [] we enrolled patients with a diagnosis of acute ischemic stroke admitted to the Internal Medicine Department at the University of Palermo and to the Neurology Division of Azienda Ospedaliera Civico of Palermo between November 2006 and October 2008. The control subjects were patients admitted, in the same period, to our Internal Medicine Department for any cause different from acute cardiovascular and cerebrovascular events or exclusion criteria. Every subject with ischemic stroke was matched for age ( \pm 3 years), sex, and cardiovascular risk factor prevalence with one control subject.

In our previous work [120] we compared only lacunar stroke with non-lacunar strokes (LAAS, CEI and ODE), but in this previous work the small number of subjects enrolled $(n=60)$ did not allow us to analyse immuno-inflammatory profile of the acute phase of ischemic stroke of each diagnostic subtype, due to the fact that when divided into subgroups the numbers become even smaller, enhancing the possibility of chance skewing that could become significant. Nevertheless, in our present study we present data of new enrolled 107 (according sample size calculation) patients with ischemic stroke, so it was feasible to divide our enrolled patients into subgroups in relation to TOAST diagnostic subtype and evaluate cytokine, selectins and adhesion molecules with regard to each diagnostic subtype.

Stroke was defined by focal neurological signs or symptoms thought to be of vascular origin that persisted for N24 h, confirmed by brain $\mathrm{CT}$ and/or MRI in baseline conditions. In order to match patients with acute ischemic stroke and controls for cardiovascular risk and previous cardiovascular morbidity, controls were included if they had vascular risk factors or a history of myocardial infarction, cerebrovascular disease or peripheral vascular disease, but they were excluded if they had either current or recent (up to 6 months) cerebrovascular disease or one of the other exclusion criteria (see Exclusion criteria section). Cardiovascular risk factors were evaluated for both subjects and controls on the basis of the following criteria: type 2 diabetes was determined using a clinically based algorithm that considered age at onset, presenting weight and symptoms, family history, onset of insulin treatment, and history of ketoacidosis. Atrial fibrillation was diagnosed when present on the admission ECG. Hypertension was present when a patient had received antihypertensive treatment before admission or when hyper- 
tension was diagnosed during the hospital stay by repeated detection of blood pressure $\geq 160 / 95 \mathrm{~mm} / \mathrm{Hg}$. Hypercholesterolemia was defined as total serum cholesterol $\geq 200 \mathrm{mg} / \mathrm{dl}$ or on the basis of a clinical history of hypercholesterolemia or statin treatment.

Patients with inflammatory or infectious diseases, cancer, haematological diseases and severe renal or liver failure, as well as those who were under treatment with anti-inflammatory drugs were excluded. We also excluded patients with fever, recent venous thromboembolism and rheumatologic disease.

The type of acute ischemic stroke was classified according to the TOAST classification [15]: 1) Large Artery Athero-Sclerosis (LAAS); 2) CardioEmbolic Infarct (CEI); 3) LACunar infarct (LAC); 4) stroke of Other Determined Etiology (ODE); 5) stroke of UnDetermined Etiology (UDE).

All the ischemic stroke patients underwent: medical history with recording of potential stroke risk factors, blood and coagulation tests, 12-lead ECG, 24 h electrocardiography monitoring, transthoracic echocardiography, carotid ultrasound, brain CT or MRI at admission (repeated between the third and the seventh days of stroke onset). Blood samples were obtained in the non-fasting state. After $10 \mathrm{~min}$ of rest in the supine position, vital signs were recorded and blood samples were collected from the antecubital vein. EDTAanticoagulated peripheral blood was drawn from each patient within $12 \mathrm{~h}$ from symptom onset. Serum and plasma were immediately separated by centrifugation and stored in aliquots at $-80{ }^{\circ} \mathrm{C}$ until analysis.

We evaluated plasma levels of IL-1 $\beta$, TNF- $\alpha$, IL- 6 and IL-10, E-selectin, P-selectin, sICAM-1 and sVCAM-1 as markers of immunoinflammatory activation, VWF plasma levels as a marker of endothelial dysfunction, TPA-antigen and PAI-1 plasma levels as thrombotic/fibrinolytic markers.

We enrolled 107 subjects with acute ischemic stroke and 102 matched controls (without ischemic stroke). Among stroke patients, according to TOAST Classification, 41 were classified as LAAS, 32 as lacunar, 31 as CEI and 3 as ODE. Stroke patients in comparison with controls showed significantly higher median plasma levels of IL1- $\beta$, IL-6, TNF- $\alpha$, E-Selectin P-Selectin VCAM-1 and ICAM1. Interestingly, patients with cardioembolic subtype showed a significantly lower median Scandinavian Stroke Scale Score [29 (2339)] compared to other subtypes, whereas stroke patients classified as lacunar showed a significantly higher median SSS score [46. 5 (47-50.7)].

Patients with ischemic stroke classified as cardioembolic (CEI) showed, compared to other subtypes, significantly higher median plasma levels of TNF- $\alpha$ [37. 2 (21. 2-48)); pb0. 0001], IL-6 [12 (6. 5-18); $\mathrm{p}=0.0029$ ], IL-1 $\beta$ [13. 5 (7-17); pb0. 0001], whereas plasma levels of Von Willebrand factor in patients with cardioembolic subtype were higher in comparison with other subtypes but were only near a statistical significance $[12(6-13)(\mathrm{p}=0$. 0053). No significant difference in terms of plasma levels of thrombotic/fibrinolytic markers was observed between each stroke subtype Furthermore, stroke patients classified as lacunar showed, compared to other subtypes, significantly lower median plasma levels of TNF- $\alpha$ [18. 4 (11-23) pg/ml; pb0. 0001]; IL-6 [5 (2-8) $\mathrm{pg} / \mathrm{ml} ; \mathrm{p}=0.0029)], \mathrm{IL}-1 \beta$ [4 (2-10) pb0. 0001$)]$, whereas plasma levels of Von Willebrand factor in [5 (2-9) ng/ml] patients with lacunar subtype were lower in comparison with other subtypes, but were only nearly at statistical significance.

Interestingly, among patients with lacunar stroke, no significant difference in median plasma levels of TNF- $\alpha$, IL- 6 and IL- $1 \beta$ was observed between subjects with and without a lacunar detectable lesion on neuroimaging (respectively $16.8 \mathrm{pg} / \mathrm{ml}, 9.5 \mathrm{pg} / \mathrm{ml}$ and 7 $\mathrm{pg} / \mathrm{ml}$ vs $17.2, \mathrm{pg} / \mathrm{ml}, 10 \mathrm{pg} / \mathrm{ml}, 6.8 \mathrm{pg} / \mathrm{ml} ; \mathrm{p}=0.87)$. Multiple linear regression performed to investigate associations between some relevant variables and SSS score as a dependent variable showed a significant association between SSS score at admission and diagnostic subtype: lacunar $(b=3.71 ; p=0.029)$ ] or [cardioembolic $(b=-8$. 011; $\mathrm{p}=0.0007)]$ and some inflammatory variable [TNF- $\alpha(b=-0.010 ;$ pb0. 0001) or IL-6 (b=-0. 068; pb0. 0001)1].

Our findings showed that patients with acute ischemic stroke compared to controls without stroke have significantly higher median plasma levels of some immune-inflammatory markers and significative difference in comparison with controls with regard of plasma levels of some thrombotic/fibrinolytic markers such as PAI1 and TPA.

We also showed that subjects with cardioembolic TOAST subtype showed significantly higher plasma levels of TNF- $\alpha$, IL-6 and IL-1 $\beta$, whereas lacunar subtype showed significantly lower plasma levels of these cytokines, whereas no significative difference was observed among each TOAST subtype of stroke with regard of thrombotic/fibrinolytic marker plasma levels. Our study further supports the hypothesis that inflammation may have an important role in the pathogenesis of ischemic strokes, but it also suggests the hypothesis of a peculiar immuno-inflammatory pattern as a pathogenic background of every subtype of ischemic stroke.

Cerebral ischemia initiates a complex cascade of events at genomic, molecular, and cellular levels, and inflammation is important in this cascade, both in the Central Nervous System (CNS) and in the periphery. The entire spectrum of inflammatory processes is likely to act in concert but cytokines are important mediators of stroke induced immunological/inflammatory reactions, which contribute to brain infarct progression as well as to the disease severity and outcome [119]. Indeed cytokines may have various functions during cerebral ischemia, on the one hand, they can attract leukocytes and stimulate the synthesis of adhesion molecules in leukocytes, endothelial cells, and other cells, thus promoting the inflammatory response of damaged cerebral tissue. On the other, they can facilitate thrombogenesis by increasing the levels of plasminogenactivating inhibitor-1 tissue factor (PAI-1), and platelet activating factor and by inhibiting tissue plasminogen activator and protein [121]. Clinical studies [122, 123] have reported increased levels of proinflammatory cytokines and adhesion molecules in the peripheral blood and cerebrospinal fluid (CSF) of patients with ischemic stroke. Among the cytokines involved in the pathogenesis of ischemic stroke, TNF- $\alpha$ is activated in experimental ischemia at both the mRNA and protein levels.

Furthermore, increased levels of cytokines such as interleukin IL- $1 \beta$, tumor necrosis factor- $\alpha$ (TNF- $\alpha$ ), and IL- 6 , as well as adhesion molecules such as intercellular adhesion molecule-1 (ICAM1), have been observed after experimental brain ischemia.

A question that arises is, does a relationship between differential immuno-inflammatory activation of the acute phase in each TOAST subtype and stroke prognosis? In our study patients with cardioembolic strokes, in comparison with other subtypes, showed a higher degree of acute neurological deficit at admission evaluated by SSS scale and a higher degree of immuno-inflammatory activation of the acute phase. On the other hand in patients with lacunar stroke, compared to other subtypes, we observed a lower grade of acute neurological deficit at admission evaluated by SSS scale and a lower degree of immuno-inflammatory activation of the acute phase.

Moreover, regression analysis of stroke subtype (lacunar or cardioembolic) and plasma levels of IL- 6 and TNF- $\alpha$ were the variables more strictly associated with clinical outcome in terms of SSS score, with cardioembolic subtype and higher plasma levels of these cytokines associated with a poor clinical outcome and lacunar subtype, and lower plasma levels of cytokines associated with a better outcome.

In our study, logistic regression analysis showed a significant relationship between some inflammatory markers (TNF- $\alpha$, IL-6) and demographic variables (age), diagnostic subtype (lacunar or cardioembolic) and SSS score. Age is a widely described outcome 
predictor, but particularly interesting is the apparent link between diagnostic subtype, immuno-inflammatory activation of the acute phase and prognosis. In our study cardioembolic strokes showed the highest grade of immuno-inflammatory activation of the acute phase and the lowest SSS score as expression of a highest neurological deficit of the acute phase. In general, cardioembolic strokes have a worse prognosis and produce larger and more disabling strokes than other ischemic stroke subtypes [124, 125]. Infarct size could represent a possible factor influencing plasma levels of immuno-inflammatory markers. Although this issue is not always investigated and confirmed [126, 127], it is conceivable that other factors could represent an additional factor that influence immunoinflammatory activation after acute ischemic stroke. Our patients with lacunar stroke with no lesion detectable on brain CT or MRI do not show any significant difference with regard to cytokine plasma levels of the acute phase compared to patients with lacunar lesions (CT or MRI detectable). So beyond a possible direct relationship between infarct size and cytokine levels, infarct site could also have an additional weight on immuno-inflammatory activation of the acute phase. Interestingly, in our patients most of the lacunar strokes had a subcortical location, whereas most of the cardioembolic strokes had a cortical location. In particular, in our patients with lacunar stroke with a sub-cortical location, most were detected in the basal ganglia and thalamus and only a minority in the corona radiata. Sub-cortical infarcts have been classified into two pathogenically different types: striatocapsular infarcts and terminal supply area infarcts [128] fected structures of large striatocapsular infarcts include the caudate head, anterior limb of the internal capsule, and the putamen corresponding to the territories of the lenticulostriate arteries $[129,130]$. On the other hand, terminal supply area infarcts are not located in the basal ganglia themselves but in the more rostral periventricular and supraventricular white matter of the corona radiate and the centrum semiovale [130]. White matter infarcts in these two regions, resulting from relatively reduced perfusion in two or three neighboring arterial supply areas, have been called together "terminal supply area infarcts." However, they occur simultaneously in some patients and separately in others. Zulch's work [131] has shown that periventricular white matter of the corona radiata is the watershed zone between the deep and superficial territories of the MCA. population of patients with atrial fibrillation (AF) is growing markedly with the increasing number of the elderly [132] . In general, the prognosis of cardioembolic stroke tends to be poor as a result of obstruction of the major brain arteries by large fibrin-rich thrombi to produce large brain infarcts [133]. Cerebral ischaemia initiates a complex cascade of events at genomic, molecular, and cellular levels, and inflammation is important in this cascade, both in the central nervous system (CNS) and in the periphery $[134,135]$. Furthermore, recent research points to the role of chemokines, normally key factors in inflammation, in thrombogenesis [136], probably also explaining hyper-responsiveness of platelets in ischaemic stroke [137]. Recently our group reported that lacunar strokes compared to nonlacunar ones exhibited significantly lower plasma levels of tumour necrosis factor (TNF)- $\alpha$ and interleukin (IL)1- $\beta$, P-selectin and intercellular adhesion molecule 1 (ICAM-1) 24-72 hours (h) and 710 days after stroke onset [138]. One previous study examined inflammatory pathways in lacunar study [139], whereas no study, to our knowledge, evaluated inflammatory pathways in acute cardioembolic strokes. Furthermore, few studies have examined the relationship between blood levels of inflammatory biomarkers and stroke outcome [118, 119], but data on a relation between inflammation parameters and diagnostic subtype remain scarce.

We conducted another study [138] to evaluate cytokines, selectins and adhesion molecule plasma levels in patients with acute ischaemic stroke in relation to diagnostic subtype and to evaluate the relationship between clinical, laboratory and immunoinflammatory variables and acute neurological deficit of cardioembolic strokes in comparison with other stroke subtypes.
We chose to evaluate these biomarkers because acute ischaemic stroke has been associated with serum elevations of a series of immuno-inflammatory variables such as TNF- $\alpha$, IL- 6 , IL1- $\beta$, selectins adhesion molecules and of markers of impaired haemostasis and thrombosis. Thus, it appears that inflammation, mediated by both molecular components, including cytokines, selectins and adhesion molecules and cellular components, such as leukocytes and microglia may be important in the mechanisms of cerebral injury and repair.

We enrolled all consecutive patients with a diagnosis of acute ischaemic stroke admitted to the Internal Medicine and CardioAngiology Department at the University of Palermo (Italy) between November 2002 and January 2006. Controls were patients admitted, in the same period, to our department for any cause other than acute cardiovascular and cerebro-vascular events or exclusion criteria (see exclusion criteria section).

Stroke was defined by focal neurological signs or symptoms thought to be of vascular origin that persisted for $>24 \mathrm{~h}$ confirmed by brain computed tomography (CT) and /or magnetic resonance imaging (MRI) scan in baseline conditions and brain CT with contrast medium after 48-72 h (19).

In order to also match patients with acute ischaemic stroke and controls for cardiovascular risk and previous cardiovascular morbidity, controls were included if they had vascular risk factors or a history of myocardial infarction or cerebrovascular disease or peripheral vascular disease, but they were excluded if they had either current or recent (up to six months) cerebro-vascular disease or one of the exclusion criteria.

We enrolled 156 subjects with acute ischaemic stroke and 123 matched controls (without ischaemic stroke). Among stroke patients, according to TOAST Classification, 50 were classified as LAAS, 46 as lacunar, 20 as CEI and 4 as ODE.

Stroke patients compared to controls showed significantly higher median plasma levels of IL1- $\beta$, IL- 6 , TNF- $\alpha$, E-selectin Pselectin V-CAM-1 and ICAM-1. The mean ischaemic lesion volume on brain CT was $1077.7 \mathrm{~mm} 3$. The brain lesion mean volume was larger in patients with cardio-embolic stroke infarction (mean $12273 \mathrm{~mm} 3$ ) than in those with atherosclerotic lesions (mean 11894 mm3) and lacunar infarct (mean $9894 \mathrm{~mm} 3$ ).

Interestingly, patients with cardio-embolic subtype showed a significantly lower median SSS score [29 (11-34)] compared to other subtypes, whereas stroke patients classified as lacunar showed a significantly higher median SSS score [49. 5 (47-50. 7)].

Patients with ischaemic stroke classified as cardio-embolic (CEI), compared to other subtypes, showed significantly higher median plasma levels of TNF- $\alpha$ [38. 5 (22. 2-46) $\mathrm{pg} / \mathrm{ml} ; \mathrm{p}<0$. 0001], IL-6 [11 (5. 5-19) pg/ml; p=0. 0029], IL1- $\beta$ [11. 5 (8-13) $\mathrm{pg} / \mathrm{ml} ; \mathrm{p}<0.0001]$, whereas plasma levels of VWF in patients with cardio-embolic subtype were higher compared to other subtypes but were only near statistical significance $[10(5-12) \mathrm{ng} / \mathrm{ml}(\mathrm{p}=0$. 0053)]. Furthermore, stroke patients classified as lacunar showed, compared to other subtypes, significantly lower median plasma levels of TNF- $\alpha$ [19. 4 (9-23) pg/ml; p< 0. 0001]; IL-6 [(4 (2-9) $\mathrm{pg} / \mathrm{ml} ; \mathrm{p}=0.0029)], \mathrm{IL} 1-\beta[6(10-3) \mathrm{pg} / \mathrm{ml} \mathrm{p}<0.0001)]$ whereas plasma levels of VWF [6 (3-8) $\mathrm{ng} / \mathrm{ml}(0.0057)]$ in patients with lacunar subtype were lower in comparison with other subtypes, but were only near statistical significance. Patients with LAAS subtype of acute ischaemic stroke compared with subjects with cardioembolic subtype had a lower mean brain infarct volume (11894 $\mathrm{mm} 3$ vs. $12273 \mathrm{~mm} 3$ ) and significantly lower median plasma values of TNF- $\alpha$ [27. 5 (13. 4-40. 5) pg/ml vs. 38.5 (22. 2-46) pg/ml] , IL-6 [8 (4-12) pg/ ml vs. 11 (5. 5-19)pg/ml], IL1- $\beta$ (9 (5-10, 5) $\mathrm{pg} / \mathrm{ml}$ vs. $11.5(8-13) \mathrm{pg} / \mathrm{ml}$. No significative difference was observed between each TOAST subtype with regard of ICAM-1, VCAM-1, E-selectin, P-selectin, VWF, PAI-1 and TPA plasma levels. 
Nevertheless comparing only the seven patients among LAAS and CEI strokes, that were comparable on the basis of matched mean brain infarct volume $(11350 \mathrm{~mm} 3$ vs. $11455 \mathrm{~mm} 3)$, patients with cardio-embolic stroke showed similar median plasma levels of TNF- $\alpha$ [26. 2 (11. 4-37. 5) pg/ml vs. 26.7 (11. 9-38. 1) pg/ml, IL-6 [7 (3-11) pg/ml vs. 7.5 (5. 5-13) pg/ml], IL1- $\beta$ [10 (5-11. 5) pg/ml vs. $11.5(8-13) \mathrm{pg} / \mathrm{ml}]$ and similar mean SSS score (34 vs. 33).

Infarct sites at brain CT in relation to TOAST diagnostic subtype. Interestingly, among patients with lacunar stroke no significant difference in median plasma levels of TNF- $\alpha$, IL- 6 and IL1- $\beta$ was observed between subjects with and without a lacunar detectable lesion on neuroimaging $(19.8 \mathrm{pg} / \mathrm{ml}, 9 \mathrm{pg} / \mathrm{ml}$ and $6 \mathrm{pg} / \mathrm{ml}$ vs. 18. $9, \mathrm{pg} / \mathrm{ml}, 10 \mathrm{pg} / \mathrm{ml}, 5.5 \mathrm{pg} / \mathrm{ml}$, respectively; $\mathrm{p}=0.87)$.

Multiple linear regression performed to investigate associations between some relevant variables and SSS score as a dependent variable showed a significant association between SSS score at admission and diagnostic subtype: lacunar $(b=3.206 ; p=0.0338)]$ or [cardio-embolic $(b=-7.819 ; p=0.0006)$ ], infarct volume of cardio-embolic strokes and some inflammatory variable $[\mathrm{TNF}-\alpha(\mathrm{b}$ $=-0.013 ; p<0.0001)$ or IL-6 $(b=-0.074 ; p<0.000) 1]$.

Our findings show that among patients with acute ischaemic stroke, patients with cardio-embolic subtype showed significantly higher plasma levels of TNF- $\alpha$, IL-6 and IL1- $\beta$, whereas lacunar subtype showed significantly lower plasma levels of these cytokines. Our study further supports the hypothesis that inflammation may have an important role in the pathogenesis of ischaemic strokes, but our findings also suggest a peculiar immunoinflammatory pattern in each subtype of acute ischaemic stroke.

The first cells responding to brain ischaemia are glial cells, particularly microglia, with transcription of early pro-inflammatory cytokines such as IL1- $\beta$ and TNF- $\alpha$ able to activate additional inflammatory pathways leading to induction of nitric oxide, adhesion molecules and IL-6 [118, 119].

Patients with cardio-embolic strokes, in comparison with other subtypes, showed a higher degree of acute neurological deficit at admission evaluated by SSS scale and a higher degree of immunoinflammatory activation of the acute phase. On the other hand in patients with lacunar stroke, compared to other subtypes, we observed a lower degree of acute neurological deficit at admission evaluated by SSS scale and a lower degree of immunoinflammatory activation of the acute phase. Nevertheless, patients with LAAS stroke subtype showed higher median plasma levels of some immuno-inflammatory markers (TNF- $\alpha$, IL-6 and IL1- $\beta$ ) compared to lacunar subtype, but lower median plasma levels of these biomarkers compared to the CEI subtype .

Our finding concerning higher plasma levels of immunoinflammatory markers in patients with cardio-embolic stroke underlines the role of inflammation in the pathogenesis of cerebral cardio-embolism. Evidence is growing that inflammation may be associated with AF [139] and it may underlie pathogenesis of the arrhythmia [140]. Inflammation leads to the adhesion, recruitment and trans-endothelial migration of leucocytes into the intimae, which is mediated by adhesion molecules on the endothelial cell membrane; leading to the initial capture and rolling of leucocytes along the endothelium [141].

Patients with cardio-embolic stroke showed a significantly lower median SSS score compared to patients with other subtypes of stroke, whereas patients with lacunar stroke showed a significantly higher SSS score compared to other clinical subtypes of stroke. Moreover, on regression analysis stroke subtype (lacunar or cardio-embolic), infarct volume of cardio-embolic stroke and plasma levels of IL- 6 and TNF- $\alpha$ were the variables most strictly associated with acute neurological deficit evaluated by SSS score, with cardio-embolic subtype and higher plasma levels of these cytokines associated with a poor clinical presentation (lower SSS score) and lacunar subtype and lower plasma levels of cytokines associated with a better clinical presentation (higher SSS score).

Infarct size could represent a possible factor influencing immuno-inflammatory marker plasma levels, although this issue is not always confirmed [141-142], but it is conceivable that other factors could represent an additional factor that influence immunoinflammatory activation after acute ischaemic stroke. Indeed ischaemic brain injury secondary to arterial occlusion is characterized by acute local inflammation, which involves accumulation of poly-morphonuclear neutrophils (PMN). Factors that influence the recruitment of PMN on infarct site could modify local cytokine production. Identification of factors involved in the selective recruitment and accumulation of inflammatory cells into ischaemic brain tissue is likely to expand our understanding of the mechanisms that result in transient or permanent neurological deficits, but the mechanisms behind the entry of leukocytes to sites of ischaemia are not well understood.

Nevertheless in $25 \%$ to $40 \%$ of patients with ischaemic stroke, neurological symptoms progress during the initial hours [143-144]. Although several clinical, radiological, and biochemical factors have been associated with early neurological deterioration, most of them have a low predictor value [145-146]. Regarding our patients with lacunar stroke, about $20 \%$ of our patients did not have visible infarction on CT or MRI, which is similar to the results of previous studies [147, 148].

Our patients with lacunar stroke with no lesion detectable on brain CT or MRI do not show any significant difference with regard to cytokine plasma levels of the acute phase compared to patients with lacunar lesion (CT- or MRI-detectable). So beyond a possible direct relationship between infarct size and cytokine levels, infarct site could also have an additional role in immunoinflammatory activation of the acute phase. Interestingly, in our patients most of the lacunar strokes had a sub-cortical location, whereas most of the cardio-embolic strokes had a cortical location. In particular in our patients with lacunar stroke and a sub-cortical location, most were located in basal ganglia and thalamus and only a minority in corona radiata. Subcortical infarcts have been classified into two pathogenically different types: striatocapsular infarcts and terminal supply area infarcts [147, 148].

Arterial stiffness is increasingly recognized as an important determinant of cardiovascular risk [151-152] and it may be directly involved in the process of atherosclerosis [152]. Factors underlying increased arterial stiffness are not well understood, but both functional and structural alterations in the vessel wall are thought to be important. Clinical studies evaluated arterial stiffness in patients with cardiovascular risk factors such as hypertension [153-156], diabetes [157], and end-stage renal disease [158], whereas only one study [159] analyzed pulse wave velocity (PWV) in stroke patients although, in this study, they did not have controls for comparison of their findings.

No study has evaluated both arterial stiffness indexes (pulse wave velocity and augmentation index) in patients with an acute cardiovascular or cerebrovascular event.

On this basis we conducted a study [160] to evaluate arterial stiffness indexes in subjects with acute ischemic stroke and to evaluate the relationship between these indexes and other clinical and laboratory variables.

The hypothesis of our study was that in acute phase of ischemic stroke values of markers of arterial stiffness could be increased compared to control subjects without acute ischemic stroke, because immuno-inflammatory activation, endothelial dysfunction and other potentially toxic events characteristic of the acute phase of stroke. Further assumption was that, in relation to the different pathogenesis of each TOAST subtype, markers of arterial stiffness could have different values in different TOAST subtype. 
We enrolled all consecutive patients with a diagnosis of acute ischemic stroke admitted to the Internal Medicine Department at the University of Palermo between November 2006 and January 2009, and hospitalized control patients without a diagnosis of acute ischemic stroke. Control subjects were patients admitted, in the same period, to our Internal Medicine Department for any cause other than acute cardiovascular and cerebrovascular events.

Inclusion criteria were: a diagnosis of acute ischemic stroke defined by focal neurological signs or symptoms thought to be of vascular origin that persisted for $>24 \mathrm{~h}$, confirmed by brain CT and/or MRI in baseline conditions and brain CT with contrast medium after 48-72 h. Exclusion criteria were: age $<18$ years and $>80$ years, acute myocardial infarction (AMI), haemorrhagic stroke.

In order to match patients with acute ischemic stroke and controls also for cardiovascular risk and previous cardiovascular morbidity, controls were included if they had vascular risk factors or a history of myocardial infarction or cerebrovascular disease or peripheral vascular disease, but they were excluded if they had either current or recent (up to six months) cerebrovascular disease or one of the exclusion criteria (see above).

The type of acute ischemic stroke was classified according to the TOAST classification [15]: (1) Large-Artery AtheroSclerosis (LAAS); (2) Cardio-Embolic Infarct (CEI); (3) LACunar infarct (LAC); (4) stroke of Other Determined Etiology (ODE); (5) stroke of UnDetermined Etiology (UDE). We enrolled 107 patients with acute ischemic stroke and 102 control subjects matched for age, sex, cardiovascular risk factors and previous cardiovascular morbidity.

According to the TOAST criteria, the etiology of stroke was large-artery atherosclerosis (LAAS) in $41(38.31 \%)$ patients, cardioembolism (CEI) in 31 (28. 97\%) patients, lacunar stroke in 32 (29. 92\%) patients whereas $3(2.80 \%)$ subjects were classified as ODE. After adjustment for glucose, cholesterol and triglyceride blood levels stroke patients, compared to subjects without acute ischemic stroke, showed a higher mean Augmentation Index (Aix) $(103 \pm 3$. $5 \%$ vs. $99 \pm 4.6 \%)$ and PWV (11. $8 \pm 3.3 \mathrm{~m} / \mathrm{s}$ vs10. $02 \pm 2.29 \mathrm{~m} / \mathrm{s})$. After adjustment for SBP/DBP, MAP, Diabetes, Hypertension, Hypercholesterolaemia, Previous TIA, Previous stroke, Glucose blood levels, Total cholesterol blood levels LDL cholesterol, Triglyceride blood levels, Microalbuminuria, Augmentation Index (Aix) and PWV values in lacunar subjects $(112 \pm 5.5 \% ; 11.98 \pm 2$. $445 \mathrm{~m} / \mathrm{s}$ ) were significantly higher compared to values observed in LAAS $(108 \pm 3.5 \%$; $5.80 \pm 0.445 \mathrm{~m} / \mathrm{s})$, CEI $(104 \pm 3.5 \% ; 10.40 \pm 2$. $445 \mathrm{~m} / \mathrm{s})$ and ODE $(105 \pm 3.5 \% ; 11.48 \pm 1.345 \mathrm{~m} / \mathrm{s})$ subtypes.

Relationship between PWV and clinical and laboratory variables in stroke patients PWV was significantly and positively related with body mass index (BMI) $(r=0.416 ; \mathrm{p}<0.001)$, age $(\mathrm{r}=$ $0.403 ; \mathrm{p}<0.001), \operatorname{SBP}(0.493 ; \mathrm{p}<0.001)$, mean arterial pressure (MAP) $(r=0.579 ; p<0.001)$, hypertension $(r=0.403 ; p<0$. 001), diabetes $(r=0.397 ; p<0.05)$, previous transient ischemic attack (TIA) $(\mathrm{r}=0.319 ; \mathrm{p}=0.045)$, previous stroke $(\mathrm{r}=0.380 ; \mathrm{p}$ $<0.001)$, glucose blood levels $(r=0.307 ; p=0.039)$, low density lipoprotein $(\mathrm{LDL})$ cholesterol plasma levels $(\mathrm{r}=0.355 ; \mathrm{p}<<0$. 001), total cholesterol plasma levels $(r=0.323 ; p=0.041)$, triglyceride plasma levels $(\mathrm{r}=0.318 ; \mathrm{p}<0.001)$, coronary artery disease $(\mathrm{CAD})(\mathrm{r}=0.380 ; \mathrm{p}<0.001)$, microalbuminuria $(\mathrm{r}=0$. $393 ; p<0.001)$, carotid plaque $(r=0.380 ; p<0.001)$, left ventricular hypertrophy $(\mathrm{LVH})(\mathrm{r}=0.480 ; \mathrm{p}<0.001)$, previous brain infarct at neuroimage $(r=0.390 ; p=<0.001)$, white matter hyperintensity lesions (WMHLs) $(r=0.340 ; \mathrm{p}<0$. 05). Relationship between Aix and clinical and laboratory variables in stroke patients The Aix was significantly related to BMI $(r=0.40 ; p<0.001)$, Age $(r=0.41 ; p<0.001)$, SBP $(r=0.43 ; p<0.001)$, diastolic blood pressure (DBP) $(r=0.43 ; p<0.001)$, MAP $(r=0.427 ; p<$ $0.001)$, hypertension $(\mathrm{r}=0.431 ; \mathrm{p}<0.001)$, microalbuminuria $(\mathrm{r}=$ $0.305 ; \mathrm{p}<0.001)$, carotid plaque $(0.378 ; \mathrm{p}<0.001), \mathrm{LVH}(\mathrm{r}=0$.
399; $\mathrm{p}<0.05)$, white matter hyperintensity lesions WMHLs $(\mathrm{r}=$ $00.310 ; \mathrm{p}=0.038$ ).

Our study shows that patients with ischemic stroke have higher value of indexes of arterial stiffness in relation to age, sex and cardiovascular risk factor compared to control subjects and that a significant relationship exists between these indexes and some clinical and laboratory variables.

Furthermore, at intergroup analysis our findings show a significant difference among TOAST diagnostic subtype with regard to arterial stiffness indexes. Wereport that subjects with lacunar subtype show higher values of PWV and Aix compared to LAAS and CEI subtypes. Few studies examined the relationship between arterial stiffness indexes and systemic inflammation markers such as pro-inflammatory cytokines [161], whereas no study has evaluated the relationship between these indexes and immune-inflam-matory markers in patients with an acute cardiovascular or cerebrovascular event. Only one study [162] indirectly evaluated this relationship and only with regard to erythrocyte sedimentation rate (ESR).

On this basis the aim of another study by our group [163] was to evaluate the relationship between arterial stiffness and cytokine, selectin, adhesion molecule and Von Willebrand factor plasma levels in subjects with acute ischemic stroke.

We enrolled all consecutive patients with a diagnosis of acute ischemic stroke admitted to the Internal Medicine Department at the University of Palermo between November 2006 and January 2008, and hospitalized control patients without a diagnosis of acute ischemic stroke. Control subjects were patients admitted, in the same period, to our Internal Medicine Department for any cause other than acute cardiovascular and cerebrovascular events.

Carotid-femoral PWV was measured in the supine position using the automatic device (SphygmoCor version 7. 1) that measured the time delay between the rapid upstroke of the carotid and femoral artery pulse waves. The distance between the 2 arterial points was measured on the surface of the body using a tape measure. PWV was calculated as the distance traveled by the arterial pulse wave (meters) divided by the time delay between the 2 arterial points (seconds), thus expressed as meters per second.

Applanation tonometry was used to record radial artery pressure waveform continuously, and mean values of $\geq 2$ screens of pulse waves of good quality were used for analysis. On the basis of the collected data, an averaged radial pressure waveform was generated and a corresponding aortic pressure waveform and BP calculated by the validated transfer function (SphygmoCor version 7. 1). The aortic pressure waveform was used to calculate the Aix (difference in height between the first and second systolic peaks expressed as a percentage of pulse pressure (PP).

We evaluated plasma levels of C-reactive protein (CRP), Interleukin-1beta (IL-1 $\beta$ ), tumor necrosis factor-alpha (TNF- $\alpha$ ), Interleukin-6 (IL-6) and Interleukin-10 (IL-10), E-selectin, P-selectin, intercellular adhesion molecule-1 (ICAM-1), vascular cell adhesion molecule-1 (VCAM-1), as markers of immune-inflammatory activation, von Willebrand Factor (vWF) plasma levels as a marker of endothelial dysfunction, tissue plasminogen activator (TPA) and plasminogen activator inhibitor-1 (PAI-1) as marker of thrombotic/ fibrinolytic pathway.

We enrolled 107 patients with acute ischemic stroke and 107 control subjects matched for age, sex, cardiovascular risk factors and previous cardiovascular morbidity.

According to the TOAST criteria, the etiology of stroke was large-artery atherosclerosis (LAAS) in $41(38.31 \%)$ patients, cardioembolism (CEI) in $31(28.97 \%)$ patients, lacunar stroke in 32 (29. $92 \%$ ) patients whereas $3(2.80 \%)$ subjects were classified as ODE. There was a significant positive relationship, corrected for age, and gender, between PWV and CRP $(r=0.36 ; P<0$. 001), TNF- $\alpha(r=0$. 
42; $P<0.001), \operatorname{IL} 1 \beta(r=0.35 ; P<0.001), \mathrm{VWF}(r=0.46 ; P<0.001)$, and IL-6 $(r=0.21 ; P<0.05)$.

The Aix was significantly related, after correction for age and gender, to VWF $(r=0.38 ; P<0.0001)$, but not to CRP $(r=0.09 ; P=0$. 37), IL-6 ( $r=0.18 ; P<0.08)$, or TNF- $\alpha(r=0.14 ; P<0.15)$ levels.

Among Lacunar subtype PWV, after correction for age and gender, was significantly and positively related to CRP, IL-1 $\beta$, IL6 , TNF- $\alpha$ and $v W F$.

In subjects with stroke classified as LAAS, PWV was significantly and positively related to CRP, IL- $1 \beta$, IL-6, TNF- $\alpha$ but not with vWF.

In CEI subtype, PWV was significantly and positively related to TNF- $\alpha$ and vWF but not with CRP, IL-6, IL-1 $\beta$. Among Lacunar and CEI subtype Aix was positively and significantly related, after correction for age and gender, only to vWF; in LAAS subgroup this relationship was significantly weaker and only close to statistical significance.

The main our findings were that patients with acute ischemic stroke had both increased inflammatory markers and arterial stiffness markers compared to control subjects without acute ischemic stroke.

This is the first study to show in patients with acute ischemic stroke that circulating levels of some immune-inflammatory markers such as CRP, IL-6, IL-1 $\beta$, TNF- $\alpha$ and VWF are related to PWV and wave reflection. Our findings show that both aortic stiffness and wave reflection are related to the degree of systemic inflammation in stroke subjects, suggesting that circulating inflammation mediators such as CRP and some pro-inflammatory cytokines can influence the stiffness of vessels distant to those involved in the disease process itself. An immune-inflammatory cascade occurs after an acute ischemic stroke $[164,165]$ so on this basis cytokine levels that we reported and measured $72 \mathrm{hr}$ from symptom onset express an acute rise of these inflammatory markers.

On this basis it is possible that observed differences with regard to the relationship between arterial stiffness indexes and inflammatory markers could be related to differences in immuneinflammatory activation of the acute phase of each TOAST diagnostic subtype.

Our finding concerning some differences in the observed correlation between immune-inflammatory markers and arterial stiffness indexes in relation to each TOAST subtype of stroke might also indicate a different degree of vascular anatomy impairment or a different distribution of certain risk factors already reported as related to the inflammation markers, such as hypertension and diabetes. In fact our patients with lacunar stroke, in which both CRP, IL6 , IL-1 $\beta$, TNF- $\alpha$ and vWF are related to PWV, have an higher frequency of both hypertension and diabetes and this finding allow it to represent, in some ways the prototype of the acute cerebrovascular event in diabetic or hypertensive subjects. Furthermore the molecular events associated with remodelling of the large and medium to small arteries also have been well characterized and involve the combinatorial influences of adhesion molecules, integrins, metallo-proteinases, the renin-angiotensin axis, and inflammation on the cellular constituents (endothelial cells, vascular smooth cells, fibroblasts, and matrix components) of the vasculature [166, 167].

Biomarkers of inflammation like CRP, interleukin-6, and tumor necrosis factor- $\alpha$ have been associated positively with both indirect (e. g. brachial artery pulse pressure) $[167,168]$ and direct measures of arterial stiffness in previous studies in apparently healthy individuals, [169] in specific patient groups (e. g. patients with hypertension) [170], and in community-based samples [171, 172, 173]. Most of these studies had modest sample sizes, but no study evaluated the relationship between stiffness indexes and immuneinflammatory markers in patients with acute ischemic stroke or other acute cardiovascular event, so our findings appear novel. Previous studies have shown that exogenously administered cytokines (specifically IL-1 $\beta$ ) cause a NO-mediated basal vasodilation in human veins by inducing the constitutively expressed endothelial nitric oxide (NO)-synthase [174]. Nevertheless in acute ischemic stroke excitotoxic or ischemic conditions excessively activate nNOS, resulting in concentrations of NO that are toxic to surrounding neurons [175]. But what are extra-cerebral large artery levels of NO in ischemic stroke subjects? No study to our knowledge analyzed this issue

Another possible limitation because of technical limitations with regard of Aix evaluation is that we recruited in patients with cardioembolic subtype of stroke patients subjects with atrial fibrillation. However, other studies that have assessed the markers of arterial stiffness in patients with atrial fibrillation, have evaluated both PWV and Aix [176, 177].

Maybe an acute NO depletion role is not directly presumable to explain an acute rise in arterial stiffness in acute stroke patients. For this series of limitations whether our findings can be extended to a chronic situation is a matter of discussion that future studies should be clarify evaluating arterial stiffness indexes change in subjects at cerebrovascular risk before and after an acute ischemic cerebrovascular event.

The arterial changes have a multitude of potential interconnected causes including endothelial dysfunction, oxidative stress, inflammation, atherosclerosis and vascular calcification. The role and contribution of the biochemical changes to arterial stiffness in the acute phase ischemic stroke is not known, but it's likely that immune-inflammatory activation, acute hyperglycemia, endothelial disfunction and other toxic effects could be responsible of a acute increase of arterial stiffness after ischemic stroke. In conclusion we reported that both aortic stiffness and wave reflection are related to the degree of systemic inflammation in stroke subjects, showing a significant relationship between immuno-inflammatory marker and PWV in LAAS and lacunar subtype of stroke and suggesting that circulating inflammation mediators after acute ischemic stroke can influence the stiffness of vessels distant to those involved in the disease process itself.

Previous studies of infection and stroke have found that most infections among stroke cases were located in the respiratory tract. [178-180] A case-control study in Germany [181] found that recent infection was associated with cardioembolic stroke. In the subgroup with febrile infection (19 of 197 strokes), almost half the patients ( 9 of 19) suffered from cardioembolic stroke. Small-vessel disease was diagnosed as the cause of cerebral ischemia only in patients without recent infection. Another study by the same researchers 17 also found recent infection significantly increased the risk of cerebrovascular ischemia from cardioembolism and tended to elevate the risk for arterioarterial embolism.

Infections may induce thrombosis and brain infarction by several mechanisms. Some mechanisms link infections with largeartery atherogenesis. Other mechanisms suggest a prothrombotic state. Infection and inflammation cause many systemic effects, including changes in lipid metabolism, platelet aggregation, lysis, blood coagulation, alterations in endothelial function, spasms in vascular smooth muscle, atheroma instability, and subsequent plaque rupture. Compared with stroke patients without infection, those with infection were found to have increased fibrin D-dimer concentrations and anticardiolipin antibody titers [182]. Likewise, infection-associated stroke patients had lower levels of activated protein $\mathrm{C}$ and ratios of active tissue plasminogen activator to plasminogen activator inhibitor- 1 and elevations in C4bp antigen levels than noninfection stroke cases or controls [183].

Although prior studies have demonstrated that $25 \%$ to $35 \%$ of stroke patients have had a recent infection, the role of infection as a 
risk factor remains unclear. Our aim was to characterize the effect of infectious/inflammatory syndromes on stroke risk.

Some authors performed [184] a case-control and crossover analyses of 233 cases and 363 controls aged 21 to 89 years performed. Cases were patients hospitalized with a first ischemic stroke at a Los Angeles, California, medical center. Controls were outpatients in the hypertension, diabetes, and general medical clinics. All subjects were administered a neurological examination, an infection/inflammation (I/I) examination, and an interview to elicit recent I/I history at baseline (within several days of stroke onset) and again approximately 2 months later. Three physicians classified subjects by the presence or absence of I/I within 1 month of the index dates, based on findings of the I/I examination, the interview report, and laboratory results.

Infections, either total or specific, were not found more frequently in cases than controls. However, patients with a recent respiratory tract infection suffered more often from large-vessel atherothromboembolic or cardioembolic stroke than did patients without infection ( $48 \%$ vs $24 \%, \mathrm{P}=0.07)$. The age- and sexadjusted relative risk estimate for these subtypes was $1.75(95 \%$ CI, 0.86 to 3. 55). The risk was notably high for those without stroke risk factors: 4.15 (95\% CI, 1. 22 to 14. 1) for normotensives, 2. 71 (95\% CI, 1.04 to 7.06$)$ for nondiabetics, and 1.74 (95\% CI, 0.74 to 4.07 ) for nonsmokers. Patients with a recent respiratory infection also had a more severe neurological deficit on admission than those without infection $(\mathrm{P}=0.05)$.

Our results suggest that respiratory tract infection may act as a trigger and increase the risk of large-vessel and/or cardioembolic ischemic stroke, especially in those without vascular risk factors Furthermore, platelet activation and its interaction with leukocytes are important in the pathophysiology of ischemic stroke. This study aimed to evaluate the value of platelet activation and plateletleukocyte interaction in different subtypes of acute, non-cardioembolic ischemic stroke.

In a study by [185] fifty-four patients with acute, non-cardioembolic ischemic stroke, including 32 small-vessel and 22 largevessel diseases, were evaluated. Platelet activation markers (CD62P, CD63, and CD40L) and platelet-leukocyte interaction were measured by flow cytometry at different time points $(<48$ hours and Days 7, 30, and 90 post-ischemic stroke). Markers were also evaluated in 28 other stroke patients in the convalescent stage (3 to 9 months after acute stroke) and in 28 control subjects. Patients with ischemic stroke had significantly increased circulating CD62P, CD63, platelet-monocyte interaction, and plateletlymphocyte interaction in the acute stage compared with the convalescent stage and control groups. Levels of CD62P and CD63 were significantly higher in the large-vessel disease group than in the small-vessel disease group, and differences in CD62P were significant even at one month. The CD40L level in the poor outcome group was significantly higher than that in the good outcome group. Stroke patients with diabetes mellitus and large-vessel disease were associated with poor outcome. Patients with large-vessel cerebral infarction elicit higher platelet activation and platelet-leukocyte interaction compared to small-vessel infarction. Further large scale trials are warranted to evaluate the relationship between platelet activation markers and outcome in stroke patients under different anti-platelet therapies, and to clarify optimal treatment.

Activation of endothelial cells and platelets is an important mediator of atherothrombosis. Markers of endothelial cell and platelet activation such as soluble adhesion molecules can be measured in plasma. Cherian et al. [186] hypothesized that patients with acute ischemic stroke would have increased blood concentrations of soluble E-selectin and von Willebrand factor (vWF), primarily reflecting activation of endothelial cells, and increased concentrations of soluble P-selectin and platelet-derived microvesicles (PDM), primarily reflecting activation of platelets, compared with healthy controls. We also hypothesized that these markers would be differentially elevated in ischemic stroke caused by large- and smallartery atherothrombosis compared with cardiogenic embolism.

We conducted a case-control study of 200 hospital-referred cases of first-ever ischemic stroke and 205 randomly selected community controls stratified by age, sex, and postal code. Using established criteria, we classified cases of stroke by etiological subtype in a blinded fashion. The prevalence of vascular risk factors and blood concentrations of E-selectin, P-selectin, vWF antigen, and PDM were determined in stroke cases within 7 days and at 3 to 6 months after stroke and in controls.

Mean blood concentrations of soluble E-selectin, P-selectin, and PDM within 7 days of stroke onset were all significantly higher in cases compared with controls. At 3 to 6 months after stroke, the mean blood concentrations of E-selectin and P-selectin fell significantly below that of controls, and PDM concentrations remained elevated. There was a strong, graded, and independent (of age, sex, and vascular risk factors) association between increasing blood concentrations of E-selectin during the acute phase and all etiological subtypes of ischemic stroke, particularly ischemic stroke caused by large-artery atherothrombosis. There was also a significant, graded, and independent association between increasing blood concentrations of vWF during the acute phase and ischemic stroke caused by large-artery atherothrombosis.

On this basis these authors demonstrated significant associations between acute elevation of blood markers of endothelial cell and platelet activation and ischemic stroke and between acute elevation of blood markers of endothelial cell activation and ischemic stroke caused by large-artery atherothrombosis. Persistent elevated blood concentrations of PDM may be a marker of increased risk of ischemic stroke.

Moreover, The CD40 ligand (CD40L) on platelets may be a critical factor to develop the acute vascular events from atheroma. Cha et al. [187] to determine the role of CD40L on platelets in atherosclerotic ischemic stroke, we serially measured the expressions of CD63, P-selectin and CD40L on platelets in patients with atherosclerotic ischemic stroke $(n=25)$ and compared them with those in patients with asymptomatic carotid stenosis $(\mathrm{n}=20)$ and in normal subjects $(\mathrm{n}=24)$.

The expressions of CD63 and P-selectin on platelets were significantly higher in patients with atherosclerotic ischemic stroke (n $=25)$ than in normal subjects $(n=24)$. The extents of surface expressions of CD63 and P-selectin on platelets showed no significant differences between atherosclerotic ischemic stroke and asymptomatic carotid stenosis. However, the CD40L expression on platelets was significantly higher in atherosclerotic ischemic stroke when compared to that in asymptomatic carotid stenosis.

In this study among the population with large artery atherosclerosis, the patients with symptomatic ischemic events showed a significantly elevated expression of CD40L on platelets compared to those without ischemic events. Therefore, the upregulation of CD40L on platelets may be a specific marker of platelet activation to provoke ischemic stroke from large artery atherosclerosis.

The sites of platelet activation in ischemic stroke are still unclear because previous reports have not identified them and various factors accompanied by stroke can activate platelets. We therefore examined the sites of platelet activation in patients with various types of ischemic stroke.

Iwamoto et al. [188] calculated The ratio of the plasma concentration of beta-thromboglobulin (BTG) in the internal jugular vein to that in the antecubital vein (BTG-B) as an indicator of platelet activation in the cerebral circulation. Plasma BTG concentration was determined in 75 control subjects and in 186 patients with various subtypes of ischemic stroke including lacunar, atherothrombotic, and cardioembolic strokes, transient ischemic attacks, and Binswanger's disease. The BTG ratio was evaluated with regard to 
subtype of stroke, time of blood sampling, size of infarct, presence of vascular lesions, and the effect of ticlopidine administration. The mean BTG ratio was increased even in the chronic phase of most subtypes of stroke with the exception of cardioembolic stroke, which exhibited a persistent elevation of BTG-B concentrations. Patients with Binswanger's disease showed a significant and frequent elevation of BTG ratio. High BTG ratios occurred in cases with vascular lesions observed on cerebral angiography. There was no correlation between the BTG ratio and infarct size. Use of ticlopidine was partially associated with a lower BTG ratio.

The findings of this study show that platelets were activated in the cerebral circulation of patients with stroke even in the chronic phase, which suggests the development of underlying vascular lesions and of thrombogenesis with or without infarction. Platelets were activated mainly within the heart in cases of cardioembolic stroke. An enhanced release reaction secondary to platelet activation was often seen in patients with Binswanger's disease, which indicates that its pathophysiology differs from that of other subtypes of stroke. In a study by Oberheiden et al. [189] the role of platelets and cellular coagulation activation in cerebral small-vessel disease (cSVD) was assessed. In 24 patients with cSVD but without established large-vessel disease, whole blood samples were obtained. Patients were divided into three subgroups (according to extent of cSVD based on morphological magnetic resonance imaging criteria. Surface expression of CD40L and CD62P on platelets, tissue-factor exposition on monocytes and platelet-monocyte aggregates were measured with flow cytometry. Plasma levels of soluble CD40L, interleukin (IL)-6 and IL-7 were assessed by ELISA. Patients with cSVD show a significantly elevated expression of platelet CD40L $(\mathrm{P}<0.001)$ and CD62P $(\mathrm{P}<0.023)$, significantly elevated amounts of platelet-monocyte aggregates $(\mathrm{P}<0$. 004), a significantly enhanced tissue-factor exposition on monocytes $(\mathrm{P}<0.019)$ and significantly lower plasma levels of IL-7 compared to 10 healthy controls. However, this platelet and monocyte activation did not correlate with the severity of cSVD. Patients with cSVD show an up-regulation of the platelet CD40L and CD62P system and an activation of cellular coagulation which might contribute to the initiation and progression of cSVD.

Nevertheless, it remains uncertain whether platelet activation in ischemic stroke is contributory or secondary to brain ischemia. The efficacy of aspirin (ASA) in stroke prevention suggests that platelet activation contributes to the occurrence of stroke. On the other hand, platelet activation may be simply a generalized consequence of cerebral ischemic damage. To examine this issue, in a study by Shah et al. [188] plasma levels of the platelet specific proteins betathromboglobulin (beta-TG) and platelet factor 4 (PF4) were measured in fifty-eight patients with various defined types of acute ischemic strokes. beta-TG was a broader indicator of platelet activation than PF4. Compared with an age-matched control group, thromboembolic and cardioembolic stroke patients had significantly elevated beta-TG levels ( $\mathrm{p}$ less than 0. 001). Also, beta-TG levels in these stroke categories were significantly higher in samples drawn within the first week after the event than in those drawn later (p less than 0. 001). In contrast, beta-TG levels in lacunar stroke patients and in most TIA patients were normal. beta-TG levels did not correlate with the volume of cerebral infarction as measured by planimetry from CT scans. Moreover, beta-TG levels in patients on chronic ASA therapy at the time of stroke did not differ from those in patients of the same diagnostic categories not taking aspirin. These data indicate that platelet activation may be important in some, but not all, subtypes of ischemic stroke and that platelet activation can occur in stroke even though the platelet cyclooxygenase pathway is suppressed.

Previous studies have shown evidence of inflammatory activation and endothelial dysfunction in individuals with lacunar infarction and WML. More recently, higher CRP levels have been associated with presence and progression of WML in a population-based sample of nondemented elderly Europeans. A significant association between higher CRP and IL6 levels and presence of silent BI was also reported in Japanese individuals. Although these studies provide support for a link between inflammatory markers and small vessel disease pathogenesis, it is yet unclear whether small vessel disease of the brain is directly and causally influenced by increased levels of inflammatory markers, or itself induces increased synthesis of inflammatory molecules, or both.

To further investigate the relationship between molecular markers of inflammation and small vessel disease of the brain, Fornage et al. [190] conducted an association study of the sequence variation in 2 inflammation genes, CRP and IL6, with presence of WML and BI among elderly participants in the Cardiovascular Health Study (CHS). Tag single nucleotide polymorphisms (SNPs) in the CRP and IL6 genes were selected from the SeattleSNPs database. In cross-sectional analyses, logistic regression models adjusting for known cardiovascular disease risk factors were constructed to assess the associations of plasma CRP and IL6 levels and common CRP and IL6 gene haplotypes with presence of WML or BI in Blacks $(\mathrm{n}=532)$ and Whites $(\mathrm{n}=2905)$.

Plasma IL6 and CRP levels were associated with presence of WML and BI in both races. In Whites, common haplotypes of the IL6 gene were significantly associated with WML and BI. The common haplotype tagged by the $-174 \mathrm{G} / \mathrm{C}$ promoter polymorphism was associated with an increased risk of WML $(\mathrm{OR}=1.14 ; 95 \% \mathrm{CI}$ : $[1.02 ; 1.28])$. The common haplotype tagged by the $-572 \mathrm{G} / \mathrm{C}$ promoter polymorphism was associated with an increased risk of BI $(\mathrm{OR}=1.57 ; 95 \%$ CI: $[1.15 ; 2.14])$. Significant associations were lacking for WML or BI with IL6 gene variation in Blacks, or with CRP gene variation in either race. This study provides evidence of a genetic basis underlying the relationship between plasma biomarkers of inflammation and small vessel disease of the brain. Further studies to elucidate the specific role of IL6 in disease pathogenesis are warranted

\section{DISCUSSION}

Within the broad large category of brain ischemia, subtypes also vary dramatically in cause: from systemic hypoperfusion to atherosclerotic large artery disease to penetrating artery disease to dissection to emboli arising from the heart valves or atria, to emboli arising from the aorta to paradoxical emboli to occlusion of cerebral and dural sinus vein to hypercoagulability. These subtypes are as different as grapes and watermelons: two substances contained within the large category of fruits [191].

Nevertheless evidence is mounting suggesting that chronic inflammation contributes to the development and consequences of cardiovascular disease. Numerous prospective studies have shown associations of serum levels of biomarkers of inflammation such as C-reactive protein (CRP), interleukin 6 (IL6), and fibrinogen with myocardial infarction, stroke, cardiovascular death, and peripheral arterial disease.

During the course of brain ischemia, inflammatory mechanisms both intrinsic to brain as well as blood are among the important mediators of focal cerebral injury. With the onset of focal brain ischemia, microglia become activated within the penumbra and thereby facilitate further local neuronal injury via pathways that include poly(ADP-ribose) polymerase- 1 activation and activation of multiple MMPs. The activation of MMPs disrupts the blood-brain barrier, alters microvascular endothelial function and impairs the functional integrity of the neurovascular unit. These events promote the entry of peripheral leukocytes into brain and expose "protected" cerebral elements to peripheral immune surveillance systems.

On this basis The onset of cerebral ischaemia triggers a cascade of proinflammatory molecular and cellular events. Clinical studies suggest that the strength of this acute response is important in early and late clinical outcomes, early clinical worsening, and extent of 
brain damage. Variables that are predictors of adverse stroke outcome include erythrocyte sedimentation rate, and levels of $\mathrm{C}$ reactive protein (CRP), interleukin- 6 , tumour necrosis factor- $\alpha$ and intercellular adhesion molecule-1. Current data indicate that inflammation serves to fuel atherosclerosis and can act as the link between atherosclerosis and atherothrombosis. Growing evidence indicates that platelets act as prominent players in the inflammatory component of these disease processes. Thus, upon activation, platelets release a series of cytokines and growth factors and express CD40 ligand, which interacts with the CD40 receptor on other major cell types involved in atherosclerosis/atherothrombosis. In healthy volunteers. Of a range of potential inflammatory biomarkers that have been reported in the literature, the best studied are CRP, TNF- $\alpha$. IL-6, IL-1 $\beta$, and other cytokines, adhesion molecules and integrins. Such biomarkers may have clinical utility for refined identification of patients at high risk for atherothrombosis in different arterial beds and for monitoring of therapeutic agents in clinical trials. If different subtypes of ischemic stroke differ in pathophysiology, clinical manifestations and possible extension of neuronal damage similar then different could be the type and degree of immunoinflammatory activation of each subtype diagnostic? Several studies also conducted by our group have been addressed to try to answer to this question showing that cardioembolic and atherosclerotic strokes in comparison with lacunar strokes show the highest degree of immunoinflammatory activation of the acute phase.

These differences in immunoinflammatory activation may probably explain differences in acute neurological deficit and disability grade at follow-up in relation of each TOAST subtype of ischemic stroke and effects of inflammation on surrogate vascular endpoints such as arterial stiffness indexes.

Maybe an acute NO depletion role is not directly presumable to explain an acute rise in arterial stiffness in acute stroke patients. For this series of limitations whether our findings can be extended to a chronic situation is a matter of discussion that future studies should be clarify evaluating arterial stiffness indexes change in subjects at cerebrovascular risk before and after an acute ischemic cerebrovascular event.

The arterial changes have a multitude of potential interconnected causes including endothelial dysfunction, oxidative stress, inflammation, atherosclerosis and vascular calcification. The role and contribution of the biochemical changes to arterial stiffness in the acute phase ischemic stroke is not known, but it's likely that immune-inflammatory activation, acute hyperglycemia, endothelial disfunction and other toxic effects could be responsible of a acute increase of arterial stiffness after ischemic stroke.

This finding may have potential therapeutic applications aimed to reducing arterial stiffness by interfering with these biochemical changes of acute phase after ischemic stroke by means neuroprotective agents like anti-inflammatory or anti-oxidizing agents or cardiovascular drug with potential pleiotropic actions such as statins, The inflammatory response in acute stroke continues to present a number of inviting therapeutic targets, but evidence of the independent contribution of inflammation over and above other risk factors is not yet ideal in any of the areas discussed. Large-scale studies of primary stroke risk that involve high-quality phenotyping of incident stroke are required. Assumptions that risk models derived from coronary heart disease apply to ischaemic stroke are likely to highlight inconsistencies due to the multiple underlying disorders that cause stroke. Acute inflammation is clearly implicated in atherosclerotic plaque instability, and in this specific subgroup of patients, imaging markers of localized inflammation may be valuable in risk stratification and clinical management. A need exists, however, for more practical techniques to achieve this.

Although initial brain inflammation can contribute to the degree of brain damage following injury, longer-term anti-inflammatory interventions to limit the degree of damage might interfere with nervous regeneration and recovery, therefore not fully understood to date appear the issue of the immune system that is not simply deleterious in the context of neurological damage after brain ischemia, Targeting inflammatory mediators such as cytokines, adhesion molecules, Toll receptors, neutrophils, macrophages and leukocyte provide opportunities to intervene with novel therapeutics in stroke. Indeed significant data demonstrate that brain inflammatory response is a contributing factor to evolving brain injury that occur following focal stroke. Along this line of research and therapeutic approach. Nevertheless, to our knowledge so far no study has examined differences in the efficacy of anti-inflammatory therapy in patients with ischemic stroke in relation to the different subtype TOAST

\section{CONFLICT OF INTEREST}

The authors confirm that this article content has no conflicts of interest.

\section{ACKNOWLEDGEMENT}

None declared.

\section{REFERENCES}

[1] Caplan, LR. Caplan's stroke, a clinical approach, 3rd ed, Butterworth-Heinemann, Boston 2000; p. 17.

[2] Vemmos KN, Bots ML, Tsibouris PK, et al. Stroke incidence and case fatality in southern Greece: the Arcadia stroke registry. Stroke 1999; 30: 363-70.

[3] Kolominsky-Rabas PL, Sarti C, Heuschmann PU, et al. A prospective community-based study of stroke in Germany: the Erlangen Stroke Project. Stroke 1998; 29: 2501-6.

[4] Carolei A, Marini C, Di Napoli M, et al. High stroke incidence in the prospective community-based L'Aquila registry (1994-1998): first year's results. Stroke 1997; 28: 2500-6.

[5] Brown RD, Whisnant JP, Sicks JD, O'Fallon WM, Wiebers DO. Stroke incidence, prevalence, and survival: secular trends in Rochester, Minnesota, through. Stroke 1989 1996; 27: 373-80.

[6] Feigin VL, Wiebers DO, Whisnant JP, O'Fallon WM. Stroke incidence and 30-day case-fatality rates in Novosibirsk, Russia, 1982 through 1992. Stroke 1995; 26: 924-9.

[7] Sarti C, Tuomilehto J, Sivenius J, et al. Declining trends in incidence, case-fatality and mortality of stroke in three geographic areas of Finland during 1983-1989: results from the FINMONICA stroke register. J Clin Epidemiol 1994; 47: 1259-69.

[8] Bonita R, Anderson CS, Broad JB, Jamrozik KD, Stewart-Wynne EG, Anderson NE. Stroke incidence and case fatality in Australasia: a comparison of the Auckland and Perth population-based stroke registers. Stroke1994; 25: 552-7.

[9] Bamford J, Sandercock P, Dennis M, Burn J, Warlow C. Classification and natural history of clinical subtypes of cerebral infarction. Lancet 1991; 337: 1521-6.

[10] Sacco SE, Whisnant JP, Broderick JP, Philips SJ, O'Fallon WM. Epidemiological characteristics of lacunar infarcts in a population. Stroke 1991; 22: 1236-41.

[11] . Koller RL: Recurrent embolic cerebral infarction and anticoagulation. Neurology 1982; 32: 283-5.

[12] Sage JI, van Uitert RL. Risk of recurrent stroke in atrial fibrillation and nonvalvular heart disease. Stroke 1983; 14: 668-76.

[13] Caplan LR, Hier DB, D'Cruz I. Cerebral embolism in the Michael Reese Stroke Registry. Stroke 1983; 14: 530-6.

[14] Bogousslavsky J, Van Melle G, Regli F: The Lausanne Stroke Registry: Analysis of 1, 000 consecutive patients with first stroke. Stroke 1988; 19: 1083-92.

[15] Adams HP Jr, Bendixen BH, Kappelle LJ, et al. Classification of subtype of acute ischemic stroke. Definitions for use in a multicenter clinical trial. TOAST. Trial of Org 10172 in Acute Stroke Treatment. Stroke 1993; 24(1): 35-41.

[16] Bogousslavsky J, Van Melle G, Regli F: The Lausanne Stroke Registry: Analysis of 1,000 consecutive patients with first stroke. Stroke 1988; 19: 1083-92.

[17] Mohr JP, Caplan LR, Melski JW, et al. The Harvard Cooperative Stroke Registry: A prospective registry. Neurology 1978; 28: 75462 
[18] Kolominsky-Rabas PL, Weber M, Gefeller O, Neundoerfer B, Heuschmann PU. Epidemiology of ischemic stroke subtypes according to TOAST criteria: incidence, recurrence, and long-term survival in ischemic stroke subtypes: a population-based study. Stroke 2001 1; 32(12): 2735-40.

[19] Petty GW, Brown RD Jr, Whisnant JP, Sicks JD, O'Fallon WM, Wiebers DO. Ischemic stroke subtypes: a population-based study of incidence and risk factors. Stroke 1999; 30(12): 2513-6.

[20] Giacomini MK. Gender and ethnic differences in hospital-based procedure utilization in California. Arch Intern Med 1996; 156: 1217-24.

[21] Gillum RF. Epidemiology of carotid endarterectomy and cerebral arteriography in the United States. Stroke 1995; 26: 1724 -8.

[22] Woo D, Gebel J, Miller R, et al. Incidence rates of first-ever ischemic stroke subtypes among blacks: a population-based study. Stroke 1999; 30: 2517-22

[23] Gorelick PB. Cerebrovascular disease in African Americans. Stroke 1998; 29: 2656-64

[24] Wityk RJ, Lehman D, Klag M, Coresh J, Ahn H, Litt B. Race and sex differences in the distribution of cerebral atherosclerosis. Stroke 1996; 27: 1974-80.

[25] Mort EA, Weissman JS, Epstein AM. Physician discretion and racial variation in the use of surgical procedures. Arch Intern Med 1994; 154: 761-7

[26] Carlisle DM, Valdez RB, Shapiro MF, Brook RH. Geographic variation in rates of selected surgical procedures within Los Angeles County. Health Serv Res 1995; 30: 27-42.

[27] Oddone EZ, Horner RD, Monger ME, Matchar DB. Racial variations in the rates of carotid angiography and endarterectomy in patients with stroke and transient ischemic attack. Arch Intern Med 1993; 153: 2781-6.

[28] Horner RD, Oddone EZ, Matchar DB. Theories explaining racial differences in the utilization of diagnostic and therapeutic procedures for cerebrovascular disease. Milbank Q 1995; 73: 443-62.

[29] Lavados PM, Sacks C, Prina L, et al. Incidence, case-fatality rate, and prognosis of ischaemic stroke subtypes in a predominantly Hispanic-Mestizo population in Iquique, Chile (PISCIS project): a community-based incidence study. Lancet Neurol 2007; 6(2): 1408 .

[30] Whiteley W, Chong WL, Sengupta A, Sandercock P. Blood markers for the prognosis of ischemic stroke: a systematic review. Stroke 2009; 40: e380-9.

[31] Castellanos M, Leira R, Tejada J, Gil-Peralta A, et al. Predictors of good outcome in medium to large spontaneous supratentorial intracerebral haemorrhages. J Neurol Neurosurg Psychiatry 2005; 76: 691-5.

[32] Castillo J, Davalos A, Alvarez-Sabin J, et al. Molecular signatures of brain injury after intracerebral hemorrhage. Neurology 2002; 58 : 624-9.

[33] Drake C, Boutin H, Jones MS, et al. Brain inflammation is induced by co-morbidities and risk factors for stroke. Brain Behav Immun 2011; 25(6): 1113-22.

[34] Brea D, Blanco M, Ramos-Cabrer P, et al. Toll-like receptors 2 and 4 in ischemic stroke: outcome and therapeutic values. J Cereb Blood Flow Metab 2011; 31(6): 1424-31.

[35] Youn CS, Choi SP, Kim SH, et al. Serum highly selective Creactive protein concentration is associated with the volume of ischemic tissue in acute ischemic stroke. Am J Emerg Med 2010 Dec 13. [Epub ahead of print

[36] Dirnagl U, Iadecola C, Moskowitz MA. Pathobiology of ischaemic stroke: an integrated view. Trends Neurosci 1999; 22: 391-7.

[37] Arvin B, Neville LF, Barone FC, Feuerstein GZ. The role of inflammation and cytokines in brain injury. Neurosci Biobehav Rev 1996; 20: 445-52.

[38] Stoll G, Jander S, Schroeter M. Inflammation and glial responses in ischemic brain lesions. Prog Neurobiol 1998; 56: 149-71.

[39] Ramsay SC, Weiller C, Myers R, Cremer JE, Luthra SK, Lammertsma AA, Frackowiak RS: Monitoring by PET of macrophage accumulation in brain after ischaemic stroke. Lancet 1992; 339: 1054-5.

[40] Enblad P, Frykholm P, Valtysson J, et al. Middle cerebral artery occlusion and reperfusion in primates monitored by microdialysis and sequential positron emission tomography. Stroke 2001; 2: 1574-80

[41] Walberer M, Rueger MA, Simard ML, et al. Dynamics of neuroinflammation in the macrosphere model of arterio-arterial embolic focal ischemia: an approximation to human stroke patterns Exp Transl Stroke Med 2010; 20; 2(1): 22.

[42] Buck BH, Liebeskind DS, Saver JL, et al. Early neutrophilia is associated with volume of ischemic tissue in acute stroke. Stroke 2008; 39(2): 355-60.

[43] Kochanek PM, Hallenbeck JM. Polymorphonuclear leukocytes and monocytes/macrophages in the pathogenesis of cerebral ischemia and stroke. Stroke 1992; 23, 1367-79.

[44] Yamasaki Y, Matsuo Y, Matsuura N, Onodera H, Itoyama Y, Kogure K. Transient increase of cytokine-induced neutrophil chemoattractant, a member of the interleu-kin- 8 family, in ischemic brain areas after focal ischemia in rats. Stroke 1995; 26, 318-22.

[45] Jander S, Pohl J, D'Urso D, Gillen C, Stoll G. Time course and localization of interleukin-10 mRNA and protein expression in autoimmune inflammation of the rat central nervous system. Am J Pathol 1998; 152: 975-82.

[46] Stoll G, Trapp BD, Grin JW. Macrophage function during Wallerian degeneration of the rat optic nerve: clearance of degenerating myelin and Ia expression. J Neurosci 1989; 9: 2327-35.

[47] Chung IL, Benveniste EN. Tumor necrosis factor preduction by astrocytes: Induction by lipopolysacharide, IFN-y, and IL- I p. J Immunol 1990; 144: 2999-3007

[48] Aloisi F, Car F, Borsellino G, et al. Production of hemolymphopoietic cytokines (IL-6, IL-8, colony-stimulating factors) by normal human astrocytes in response to IL-1/1 and tumor necrosis factor-a. J Immunol 1992; 149: 2358-66

[49] Frei K, Nohava K, Malipiero UV, Schwerdel C, Fontana A. Production of macrophage colony-stimulating factor by astrocytes and brain macrophages. J. Neuroimmunol 1992; 40: 189-98.

[50] Wang YY, Lin SY, Chuang YH, Chen CJ, Tung KC, Sheu WH. Adipose proinflammatory cytokine expression through sympathetic system is associated with hyperglycemia and insulin resistance in a rat ischemic stroke model. Am J Physiol Endocrinol Metab 2011; 300(1): E155-63.

[51] Wang Q, Tang XN, Yenari MA. The inflammatory response in stroke. J Neuroimmunol 2006; 184: 53-68.

[52] Barone FC, Schmidt DB, Hillegass LM, et al. Reperfusion increases neutrophils and leukotriene $\mathrm{B} 4$ receptor binding in rat focal ischemia. Stroke 1992; 23: 1337-47.

[53] Barone FC, Hillegass LM, Price WJ, et al. Polymorphonuclear leukocyte infiltration into cerebral focal ischemic tissue: myeloperoxidase activity assay and histologic verification. J Neurosci Res 1991; 29: 336-45.

[54] Barone FC. Ischemic stroke intervention requires mixed cellular protection of the penumbra. Curr Opin Investig Drugs 2009; 10: 220-3.

[55] Beamer NB, Coull BM, Clark WM, et al. Focal cerebral ischemia in the rat: temporal profile of neutrophil responses. Neurosci. Res. Commun 1992; 11: 179-86

[56] Qamirani E, Ren Y, Kuo L, Hein TW. C-reactive protein inhibits endothelium-dependent NO-mediated dilation in coronary arterioles by activating p38 kinase and NAD(P)H oxidase. Arterioscler Thromb Vasc Biol 2005; 25(5): 995-1001.

[57] Kuhlmann CR, Librizzi L, Closhen D, et al. Mechanisms of Creactive protein-induced blood-brain barrier disruption. Stroke 2009; 40(4): 1458-66.

[58] Welsh P, Barber M, Langhorne P, Rumley A, Lowe GD, Stott DJ Associations of inflammatory and haemostatic biomarkers with poor outcome in acute ischaemic stroke. Cerebrovasc Dis 2009; 27(3): 247-53.

[59] Shantikumar S, Grant PJ, Catto AJ, Bamford JM, Carter AM Elevated C-reactive protein and long-term mortality after ischaemic stroke: relationship with markers of endothelial cell and platelet activation. Stroke 2009; 40(3): 977-9.

[60] Ryu SR, Choi IS, Bian RX, Kim JH, Han JY, Lee SG. The effect of $\mathrm{C}$-reactive protein on functional outcome in ischemic stroke patients. Int J Neurosci 2009; 119(3): 336.

[61] Idicula TT, Brogger J, Naess H, Waje-Andreassen U, Thomassen L. Admission C-reactive protein after acute ischemic stroke is associated with stroke severity and mortality: the 'Bergen stroke study'. BMC Neurol 2009; 9: 18

[62] Minami M, Kuraishi Y, Yabuuchi K, Yamazaki K, Satoh M. Induction of interleukin-lb mRNA in rat brain after transient forebrain ischaemia. J Neurochem 1992; 58: 390-2. 
[63] Buttini H, Sauter M, Boddeke A, HWGM. Induction of interleukmb mRNA after focal cerebra1 ischaemia in the rat. Mol Brain Res 1994; 126-34.

[64] Liu T, McDonnell PC, Young PR, et al. Interleukin-1 expression in ischemic rat cortex. Stroke 1993; 24: 1746-52

[65] Liu T, Young PR, McDonnell PC, White RF, Barone FC, Feuerstein GZ. Cytokine-induced neutrophil chemoattractant mRNA expressed in cerebral hernia. Neurosc. Lett 1993; 164: 125-8.

[66] Liu T, Clark RK, McConnell PC, et al. Upregulation of intercellular adhesion molecules-1 (ICAM-1) expression in primary cultures of human microvessel endothelial cells by cytokines and lipopolysaccharide. J. Neuroimmunol 1992; 39: 1 1-21.

[67] Palladino MA, Bahjat FR, Theodorakis EA, Moldawer LL. AntiTNF-alpha therapies: the next generation. Nat Rev Drug Discov 2003; 2(9): 736-4.

[68] Tarkowski E, Rosengren L, Blomstrand C, et al. Early intrathecal production of interleukin- 6 predicts the size of brain lesion in stroke. Stroke 1995; 26: 1393-8.

[69] Matbiesen T, Anderson B, Loftenius A, von Holst H. Increased interleukin-6 levels in cerebrospinal fluid foilowing subarachnoid hemorrhage. J Neurosurg 1993; 78: 562-7.

[70] McClain CJ, Cohen, D, Phyllips R, Ott L, Young B. Increased plasma and ventricular interleukm-6 levels in head injured patients. J Lab Clin Med 1991; 118: 225-30.

[71] Liu T, Clark RK, McDonnell PC, et al. Tumor necrosis factoralpha expression in ischemic neurons. Stroke 1994; 25(7): 1481-8.

[72] Rosenberg GA, Estrada EY, Dencoff JE. Matrix metalloproteinases and TIMPs are associated with blood-brain barrier opening after reperfusion in rat brain. Stroke 1998; 29(10): 2189-95.

[73] Robertson J, Beaulieu JM, Doroudchi MM, Durham HD, Julien JP, Mushynski WE. Apoptotic death of neurons exhibiting peripherin aggregates is mediated by the proinflammatory cytokine tumor necrosis factor-alpha. J Cell Biol 2001 15; 155(2): 217-26

[74] Martin-Villalba A, Hahne M, Kleber S, et al. Therapeutic neutralization of CD95-ligand and TNF attenuates brain damage in stroke. Cell Death Differ 2001; 8(7): 679-86.

[75] Nawashiro H, Tasaki K, Ruetzler CA, Hallenbeck JM. TNF-alpha pretreatment induces protective effects against focal cerebral ischemia in mice. J Cereb Blood Flow Metab 1997; 17(5): 483-90.

[76] Arend WP, Malyak M, Guthridge CJ, Gabay C. Interleukin-1 receptor antagonist: role in biology. Annu Rev Immunol 1998; 16: $27-55$

[77] Zhang W, Smith C, Howlett C, Stanimirovic D. Inflammatory activation of human brain endothelial cells by hypoxic astrocytes in vitro is mediated by IL-1beta. J Cereb Blood Flow Metab 2000; 20(6): 967-78

[78] Nomura Y, Fujii F, Sato C, Nemoto M, Tamura M. Exchange transfusion with fluorocarbon for studying synaptically evoked optical signal in rat cortex. Brain Res Brain Res Protoc 2000; 5(1): $10-5$.

[79] Tarkowski E, Rosengren L, Blomstrand C, et al. Intrathecal release of pro- and anti-inflammatory cytokines during stroke. Clin Exp Immunol. 1997; 110(3): 492-9.

[80] Kostulas N, Kivisäkk P, Huang Y, Matusevicius D, Kostulas V. Link HIschemic stroke is associated with a systemic increase of blood mononuclear cells expressing interleukin-8 mRNA. Stroke 1998; 29(2): 462-6.

[81] Vila N, Filella X, Deulofeu R, Ascaso C, Abellana R, Chamorro A. Cytokine-induced inflammation and long-term stroke functional outcome. J Neurol Sci 1999; 162(2): 185-8.

[82] Smith CJ, Emsley HC, Vail A, et al. Variability of the systemic acute phase response after ischemic stroke. J Neurol Sci 2006; 251: 77-81.

[83] Hallenbeck JM. The many faces of tumor necrosis factor in stroke. Nat Med 2002; 8: 1363-8.

[84] Fassbender K, Rossol S, Kammer T, et al. Proinflammatory cytokines in serum of patients with acute cerebral ischemia: kinetics of secretion and relation to the extent of brain damage and outcome of disease. J Neurol Sci 1994; 22: 135-9.

[85] Beamer NB, Coull BM, Clark WM, Briley DP, Wynn M, Sexton G. Persistent inflammatory response in stroke survivors. Neurology 1997; 50: 1722-8

[86] Tarkowski E, Rosengren L, Blomstrand C, Jensen C, Ekholm S, Tarkowski A. Intrathecal expression of proteins regulating apoptosis in acute stroke. Stroke 1999; 30(2): 321-7.
[87] Vila N, Castillo J, Davalos A, Chamorro A. Proinflammatory cytokines and early neurological worsening in ischemic stroke. Stroke 2000; 31: 2325-9.

[88] Frenkel D, Huang Z, Maron R, Koldzic DN, Moskowitz MA, Weiner HL. Neuroprotection by IL-10-producing MOG CD4+ T cells following ischemic stroke. J Neurol Sci 2005; 233: 125-32.

[89] Morita Y, Takizawa S, Kamiguchi H, Uesugi T, Kawada H, Takagi S. Administration of hematopoietic cytokines increases the expression of anti-inflammatory cytokine (IL-10) mRNA in the subacute phase after stroke. Neurosci Res 2007; 58: 356-60.

[90] Kes VB, Simundic AM, Nikolac N, Topic E, Demarin V. Proinflammatory and anti-inflammatory cytokines in acute ischemic stroke and their relation to early neurological deficit and stroke out come. Clinical Biochemistry 2008; 41 1330-4.

[91] Castellanos M, Castillo J, García MM, et al. Inflammationmediated damage in progressing lacunar infarctions: a potential therapeutic target. Stroke 2002; 33(4): 982-7.

[92] Liu T, Clark RK, McDonnel PC, et al. Tumor necrosis factor-_ in ischemic neurons. Stroke1994; 25: 1481-8.

[93] Feuerstein GZ, Liu T, Barone FC. Cytokines, inflammation, and brain injury: role of tumor necrosis factor- $\alpha$. Cerebrovasc Brain Metab Rev 1994; 6: 341-60.

[94] Bitsch A, Klene W, Murtada L, Prange H, Rieckmann P. A longitudinal prospective study of soluble adhesion molecules in acute stroke. Stroke 1998; 29: 2129-35.

[95] Fassbender K, Mössner R, Motsch L, Kischka U, Grau A, Hennerici M. Circulating selectin- and immunoglobulin-type adhesion molecules in acute ischemic stroke. Stroke 1995; 26: 1361-4.

[96] Serena J, Leira R, Castillo J, Pumar JM, Castellanos M, Dávalos A. Neurological deterioration in acute lacunar infarctions: the role of excitatory and inhibitory neurotransmitters. Stroke $2001 ; 32: 1154$ 61.

[97] Greenamyre JT, Porter RHP. Anatomy and physiology of glutamate in the CNS. Neurology 1994; 44: S7-13.

[98] Sotgiu S, Zanda B, Marchetti B, et al. Inflammatory biomarkers in blood of patients with acute brain ischemia. Eur J Neurol 2006; 13(5): 505-13

[99] Stoll G, Jander S, Schroeter M. Inflammation and glial response to ischemic brain lesions. Prog. Neurobiol 1998; 56, 149-71.

[100] Feuerstein GZ, Wang X, Barone FC. Inflammatory mediators and brain injury: the role of cytokines and chemokines in stroke and CNS diseases. In: Ginsberg, MD, Bogousslavsky, J (Eds. ), Cerebrovascular Disease: Pathophysiology, Diagnosis, and Management, vol. 1. Blackwell Science, Boston, Mass1988; pp. 507-531 (10).

[101] Yamasaki Y, Matsuura N, Shozuhara H, Onodera H, Itoyama Y, Kogure K. Interleukin-1 as a pathogenetic mediator of ischemic brain damage in rats. Stroke 1995; 26: 676-81.

[102] Licata G, Tuttolomondo A, Corrao S, et al. Immunoinflammatory activation during the acute phase of lacunar and non-lacunar ischemic stroke: association with time of onset and diabetic state. Int J Immunopathol. Pharmacol 2006; 9 (3): 639-46.

[103] Del Zoppo GI, Ginis JM, Hallenback C, Iadecola X, Wang GZ. Feuerstein. Inflammation and stroke: putative role for cytokines, adhesion molecules and i-NOS in brain response to ischemia. Brain Pathol 2000; 10: 95

[104] Conolly ES. Jr,. Winfree CJ, Springer TA, et al. Cerebral protection in homozygous null ICAM-1 after middle cerebral artery occlusion: role of neutrophil adhesion in the pathogenesis of stroke. J. Clin. Invest 1996; 7: 209.

[105] Buttini MA. Sauter and H. W. Boddeke. Induction of interleukin-1 beta mRNA after focal cerebral ischaemia in the rat. Mol. Brain Res 1994; 23: 126.

[106] Eriksson CAM. Van Dam PJ. Lucassen JG. Bol Winblad B, Schultzberg M. Immunohistochemical localization of interleukin1beta, interleukin-1 receptor antagonist and interleukin-1beta converting enzyme/caspase-1 in the rat brain after peripheral administration of kainic acid. Neuroscience 1999; 93: 915.

[107] Shalaby MR, Aggarwal BB. Rinderknechte. Activation of human polymorphonuclear neutrophil functions by interferon-gamma and tumor necrosis factor. J Immunol 1985; 135: 2069.

[108] Dong JS, Fujii D, Goto T, et al. Increased expression of plasminogen activator inhibitor-1 by mediators of the acute phase response: a potential progenitor of vasculopathy in Hypertensives. Hypertens. Res 2003; 26: 723 . 
[109] Calles-Escandon J, Mirza SA, Sobel BE, Schneider DJ. Induction of hyperinsulinemia combined with hyperglycemia and hypertriglyceridemia increases plasminogen activator inhibitor 1 in blood in normal human subjects. Diabetes 1998; 47: 290.

[110] Pinto A, Tuttolomondo A, Di Raimondo D, Fernandez P. and Licata G. Cerebrovascular risk factors and clinical classification of strokes. Semin. Vasc. Med 2004; 4: 287.

[111] Tuttolomondo A, Pinto A, Di Raimondo D, Fernandez P, Licata G. Stroke patterns, etiology and prognosis in patients with diabetes mellitus. Neurology 2005; 64: 581.

[112] Hillen TC, Coshall K, Tilling A, Rudd R, Mc-Govern, Wolfe C. Cause of stroke recurrence is multifactorial. Patterns, risk factors and outcome of stroke recurrence in the South London, Stroke Register. Stroke 2003; 34: 1457.

[113] Peress NS, Kane WC. Central nervous system findings in tenth decade autopsypopulation. Prog. Brain Res 1973; 40: 473.

[114] Okada YBR, Copeland E, Mori MM, Tung WS. Thomas and G. J. Del Zoppo 1994. P-Selectin and intercellular adhesion molecule-1 expression after focal brain ischemia and reperfusion. Stroke 1994; 25: 202.

[115] Flex A, Gaetani E, Papaleo P, et al. Pola. Proinflammatory genetic profiles in subjects with history of ischemic stroke. Stroke 2004; 35: 2270 .

[116] Libby P, Ridker PM, Maseri A, Inflammation and atherosclerosis. Circulation 2002; 105(9): 1135-43.

[117] Schneider DJ, Ricci MA, Taatjes DJ, et al. Changes in arterial expression of fibrinolytic system proteins in atherogenesis. Arterioscler. Thromb. Vasc. Biol 1997; 17: 294-3301.

[118] Castellanos M, Castillo J, García M, et al. Inflammation-mediated damage in progressing lacunar infarctions. a potential therapeutic target. Stroke 2002; 33: 982-7.

[119] Vila N, Castillo J, Davalos A, Chamorro A. Proinflammatory cytokines and early neurological worsening in ischemic stroke. Stroke 2000; 31: 2325-9.

[120] Tuttolomondo A, Di Sciacca R, Di Raimondo D, et al. Plasma levels of inflammatory and thrombotic/fibrinolytic markers in acute ischemic strokes: relationship with TOAST subtype, outcome and infarct site. J Neuroimmunol 2009; 30: 84-9.

[121] Rothwell NJ, Loddick SA, Stroemer P. Interleukins and cerebral ischaemia. Int Rev Neurobiol 1997; 40: 281-98

[122] Tarkowski E, Rosengren L, Blomstrand C, et al. Early intrathecal production of interleukin-6 predicts the size of brain lesion in stroke. Stroke 1995; 26 (8): 1393-8.

[123] Intiso D, Zarrelli MM, Lagioia G, et al. Tumor necrosis factor alphaserum levels and inflammatory response in acute ischemic stroke patients. Neurol Sci 2004; 24 (6): 390-6.

[124] Hart RG, Albers G, Koudstaal P. Cardioembolic stroke. In: Ginsberg, M, Management. Blackwell Science, pp 1998: 1392-429.

[125] Lin HJ, Wolf PA, Kelly-Hayes M, et al. Stroke severity in atrial fibrillation. The Framingham Study. Stroke 1996; 27 (10): 1760-4.

[126] Fassbender K, Rossol S, Kammer T, et al. Proinflammatory cytokines in serum of patients with acute cerebral ischemia: kinetics of secretion and relation to the extent of brain-damage and outcome of disease. J Neurol Sci 1994; 122: 135-9.

[127] Carlstedt F, Lind L, Lindahl B. Proinflammatory cytokines, measured in a mixed population on arrival in the emergency department, are related to mortality and severity of disease. J Intern Med 1997; 61-365.

[128] Wodarz, R. atershed infarctions and computed tomography: a topographical study in cases with stenosis or occlusion of the carotid artery . Neuroradiology 1980; 5-248.

[129] Ringelstein EB, Zeumer H, Angelou D. The pathogenesis of strokes from internal carotid artery occlusion: diagnostic and therapeutical implications. Stroke 1983; 14, 867-875.

[130] Damasio, H. A computed tomographic guide to the identification of cerebral vascular territories. Arch. Neurol 1983; 40: 138-142.

[131] Zu“lch, K. J. U, . ber die Entstehung und Lokalisation der Hirninfarkte. Zbliz. Neurochir 1961; 21, 158-178.

[132] Kimura K, Kazui S, Minematsu K, et al. for the Japan Multicenter Stroke Investigator's Collaboration. Analysis of 16, 922 patients with acute ischaemic stroke and transient ischaemic attack in Japan. A hospital-based prospective registration study. Cerebrovasc Dis 2004; 18: 47-56.

[133] Furie KL, Homma S, Kistler JP. Cardiac diseases. In: Stroke: Pathophysiology, Diagnosis, and Management. 4th ed. Churchill Livingstone, New York, 2004: pp. 747-759.
[134] Ekdahl CT, Kokaia Z, Lindvall O. Brain inflammation and adult neurogenesis: The dual role of microglia. Neuroscience 2008; 158 : 1021-1029.

[135] Welsh P, Lowe GD, Chalmers J, et al. Associations of proinflammatory cytokines with the risk of recurrent stroke. Stroke 2008; 39: 2226-2230.

[136] Lambert MP, Sachais BS, Kowalska MA. Chemokines and thrombogenicity. Thromb Haemost 2007; 97: 722-729.

[137] Fateh-Moghadam S, Htun P, Tomandl B, et al. Hyperresponsiveness of platelets in ischaemic stroke. Thromb Haemost 2007; 97: 974-978.

[138] Licata G, Tuttolomondo A, Di Raimondo D, Corrao S, Di Sciacca $\mathrm{R}$, Pinto A. Immuno-inflammatory activation in acute cardioembolic strokes in comparison with other subtypes of ischaemic stroke. Thromb Haemost. 2009 May; 101(5): 929-37.

[139] Chung MK, Martin DO, Sprecher D, et al. C-reactive protein elevation in patients with atrial arrhythmias: inflammatory mechanisms and persistence of atrial fibrillation. Circulation 2001; 104: 28862891.

[140] Frustaci A, Chimenti C, Bellocci F, et al. Histological substrate of atrial biopsies in patients with lone atrial fibrillation. Circulation 1997; 96: 1180-1184.

[141] Aukrust P, Halvorsen B, Yndestad A, et al. Chemokines and cardiovascular risk. Arterioscler Thromb Vasc Biol 2008; 2: 1909 1919.

[142] Nakase T, Yamazaki T, Ogura N, et al. The impact of inflammation on the pathogenesis and prognosis of ischaemic stroke. $\mathrm{J} \mathrm{Neu-}$ rol Sci 2008; 271: 104-109.

[143] Rincon F, Dhamoon M, Moon Y, et al. Stroke location and association with fatal cardiac outcomes. Northern Manhattan Study (NOMAS). Stroke 2008; 39: 2425-2431.

[144] Barber M, Wright F, Stott DJ, et al. Predictors of early neurological deterioration after ischaemic stroke: a case-control study. Gerontology 2004; 50: 102-109.

[145] Serena J, Rodríguez-Yáñez M, Castellanos M. Deterioration in acute ischaemic stroke as the target for neuroprotection. Cerebrovasc Dis 2006; 21 (Suppl 2): 80-88.

[146] Lubart E, Leibovitz A, Baumoehl Y, et al. Progressing stroke with neurological deterioration in a group of Israeli elderly. Arch Gerontol Geriatr 2005; 41: 95-100.

[147] Lynch JR, Blessing R, White WD, et al. Novel diagnostic test for acute stroke. Stroke 2004; 35: 57-63.

[148] Lavados PM, Sacks C, Prina L, et al. Incidence, case-fatality rate, and prognosis of ischaemic stroke subtypes in a predominantly Hispanic-Mestizo population in Iquique, Chile (PISCIS project): a community based incidence study. Lancet Neurol 2007; 6: 140148.

[149] Vaitkevicius PV, Fleg JL, Engel JH, et al. Effects of age and aerobic capacity on arterial stiffness in healthy adults. Circulation 1993; 88: 1456-62.

[150] Boutouyrie P, Tropeano AI, Asmar R, et al. Aortic stiffness is an independent predictor of primary coronary events in hypertensive patients: a longitudinal study. Hypertension 2002; 39: 10-5.

[151] Lehmann ED, Watts GF, Gosling RG. Aortic distensibility and hypercholesterolaemia. Lancet 1992; 340: 1171-2.

[152] Lehmann ED, Gosling RG, Sonksen PH. Arterial wall compliance in diabetes. Diabet Med 1992; 9: 114-9.

[153] Muxfeldt ES, Fiszman R, Castelpoggi CH, Salles GF. Ambulatory arterial stiffness index or pulse pressure: which correlates better with arterial stiffness in resistant hypertension? Hypertens Res 2008; 31(4): 607-13.

[154] Gordin D, Rönnback M, Forsblom C, et al. Glucose variability, blood pressure and arterial stiffness in type 1 diabetes. Diabetes Res Clin Pract 2008; 80(3): e4-7.

[155] Muxfeldt ES, Fiszman R, Castelpoggi CH, Salles GF. Ambulatory arterial stiffness index or pulse pressure: which correlates better with arterial stiffness in resistant hypertension? Hypertens Res 2008; 31(4): 607-13.

[156] Gordin D, Rönnback M, Forsblom C, et al. Glucose variability, blood pressure and arterial stiffness in type 1 diabetes. Diabetes Res Clin Pract 2008; 80(3): e4-7.

[157] Rahman S, Ismail AA, Ismail SB, Naing NN, Rahman AR. Early manifestation of macrovasculopathy in newly diagnosed never treated type II diabetic patients with no traditional CVD risk factors. Diabetes Res Clin Pract 2008; 80(2): 253-8. 
[158] Blacher J, Demuth K, Guerin AP, Safar ME, Moatti N, London GM. Influence of biochemical alterations on arterial stiffness in patients with end-stage renal disease. Arterioscler Thromb Vasc Biol. 1998; 18(4): 535-41.

[159] De Silva D, Woon FP, Chen C, et al. Profile and associations of central pulse wave velocity and central pulse pressure among ischemic stroke patients. Eur J Neurol 2008; 15: 196-8.

[160] Tuttolomondo A, Di Sciacca R, Di Raimondo D, et al. Arterial stiffness indexes in acute ischemic stroke: relationship with stroke subtype. Atherosclerosis 2010; 211(1): 187-94.

[161] Mahmud A, Feely J. Arterial Stiffness Is Related to Systemic Inflammation in Essential Hypertension Hypertension 2005; 46: 1118-22.

[162] De Silva DA, Woon FP, Gan HY, et al. Arterial stiffness, metabolic syndrome and inflammation amongst Asian ischaemic stroke patients. Eur J Neurol 2008; 15(8): 872.

[163] Tuttolomondo A, Di Raimondo D, Pecoraro R, et al. Immuneinflammatory markers and arterial stiffness indexes in subjects with acute ischemic stroke. Atherosclerosis 2010; 213(1): 311-8.

[164] Zoppo G I, Ginis JM, Hallenback C, Iadecola X, Wang GZ. Feuerstein. Inflammation and stroke: putative role for cytokines, adhesion molecules and i-NOS in brain response to ischemia. Brain Pathol 2000; 10: 95-101.

[165] Sotgiu S, Zanda B, Marchetti B, et al. Inflammatory biomarkers in blood of patients with acute brain ischemia. Eur J Neurol 2006; 13(5): 505-13

[166] Intengan HD, Schiffrin EL. Vascular remodeling in hypertension: roles of apoptosis, inflammation, and fibrosis. Hypertension 2001; 38: 581-7.

[167] Brown NJ. Aldosterone and vascular inflammation. Hypertension 2008; 51: 161-7.

[168] Abramson JL, Weintraub WS, Vaccarino V. Association between pulse pressure and C-reactive protein among apparently healthy US adults. Hypertension 2002; 39: 197-202.

[169] Yasmin, McEniery CM, Wallace S, Mackenzie IS, Cockcroft JR, Wilkinson IB. C-reactive protein is associated with arterial stiffness in apparently healthy individuals. Arterioscler Thromb Vasc Biol 2004; 24: 969-74.

[170] Mahmud A, Feely J. Arterial stiffness is related to systemic inflammation in essential hypertension. Hypertension 2005; 46: 1118 -22 .

[171] Mattace-Raso FU, van der Cammen TJ, van der Meer I, et al. Creactive protein and arterial stiffness in older adults: the Rotterdam Study. Atherosclerosis 2004; 176: 111-6.

[172] Schnabel R, Larson MG, Dupuis J, et al. Relations of inflammatory biomarkers and common genetic variants with arterial stiffness and wave reflection. Hypertension. 2008; 51: 1651-7

[173] Bhagat K, Hingorani AD, Palacios M, Charles IG, Vallance P. Cytokineinduced venodilatation in humans in vivo: eNOS masquerading as iNOS. Cardiovasc Res 1999; 41: 754 -64.

[174] Lee SH, Choi S, Jung JH, Lee N. Effects of atrial fibrillation on arterial stiffness in patients with hypertension. Angiology 200; 59(4): 459-63.
[175] Samdani AF, Dawson TM, Dawson VL. Nitric oxide synthase in models of focal ischemia. Stroke 1997; 28(6): 1283-8.

[176] Kaji Y, Miyoshi T, Doi M, et al. Augmentation index is associated with B-type natriuretic peptide in patients with paroxysmal atrial fibrillation. Hypertens Res 2009; 32(7): 611-6.

[177] Syrjänen J, Valtonen VV, Iivanainen M, Kaste M, Huttunen JK. Preceding infection as an important risk factor for ischaemic brain infarction in young and middle aged patients. Br Med J 1988; 296: $1156-60$

[178] Ameriso SF, Wong VLY, Quismorio FP, Fisher M. Immunohematologic characteristics of infection-associated cerebral infarction. Stroke 1991; 22: 1004-9.

[179] Grau AJ, Buggle F, Heindl S, et al. Recent infection as a risk factor for cerebrovascular ischemia. Stroke 1995; 26: 373-9.

[180] Grau AJ, Buggle F, Steichen-Weihn C, et al. Clinical and biochemical analysis in infection-associated stroke. Stroke1995; 26 1520-6.

[181] Ameriso SF, Wong VLY, Quismorio FP, Fisher M. Immunohematologic characteristics of infection-associated cerebral infarction. Stroke 1991; 22: 1004-9.

[182] Macko RF, Ameriso SF, Gruber A, et al. Impairments of the protein $\mathrm{C}$ system and fibrinolysis in infection-associated stroke. Stroke 1996; 27: 2005-11.

[183] Paganini-Hill A, Lozano E, Fischberg G, et al. Infection and risk of ischemic stroke: differences among stroke subtypes. Stroke. 2003; 34(2): 452-7.

[184] Tsai NW, Chang WN, Shaw CF, et al. Levels and value of platelet activation markers in different subtypes of acute non-cardioembolic ischemic stroke. Thromb Res 2009; 124(2): 213-8.

[185] Cherian P, Hankey GJ, Eikelboom JW, et al. Endothelial and platelet activation in acute ischemic stroke and its etiological subtypes. Stroke 2003; 34(9): 2132-7.

[186] Cha JK, Jeong MH, Jang JY, et al. Serial measurement of surface expressions of CD63, P-selectin and CD40 ligand on platelets in atherosclerotic ischemic stroke. A possible role of CD40 ligand on platelets in atherosclerotic ischemic stroke. Cerebrovasc Dis 2003; 16(4): 376-82

[187] Iwamoto T, Kubo H, Takasaki M. Platelet activation in the cerebral circulation in different subtypes of ischemic stroke and Binswanger's disease. Stroke 1995; 26(1): 52-6.

[188] Shah AB, Beamer N, Coull BM. Enhanced in vivo platelet activation in subtypes of ischemic stroke. Stroke 1985; 16(4): 643-7.

[189] Oberheiden T, Blahak C, Nguyen XD, et al. Activation of platelets and cellular coagulation in cerebral small-vessel disease. Blood Coagul Fibrinolysis 2010; 21(8): 729-35.

[190] Fornage M, Chiang YA, O'Meara ES, et al. Biomarkers of Inflammation and MRI-Defined Small Vessel Disease of the Brain: The Cardiovascular Health Study. Stroke 2008; 39(7): 1952-9.

[191] Kumar S, Caplan LR. Why identification of stroke syndromes is still important. Curr Opin Neurol 2007; 20(1): 78-82. 